This item was submitted to Loughborough's Research Repository by the author.

Items in Figshare are protected by copyright, with all rights reserved, unless otherwise indicated.

\title{
A factorial approach to understanding the effect of inner geometry of baffled meso-scale tubes on solids suspension and axial dispersion in continuous, oscillatory liquid-solid plug flows
}

\section{PLEASE CITE THE PUBLISHED VERSION}

http://dx.doi.org/10.1016/j.cej.2016.09.013

\section{PUBLISHER}

(C) Elsevier

\section{VERSION}

AM (Accepted Manuscript)

\section{PUBLISHER STATEMENT}

This work is made available according to the conditions of the Creative Commons Attribution-NonCommercialNoDerivatives 4.0 International (CC BY-NC-ND 4.0) licence. Full details of this licence are available at: https://creativecommons.org/licenses/by-nc-nd/4.0/

\section{LICENCE}

CC BY-NC-ND 4.0

\section{REPOSITORY RECORD}

Ejim, Louisa N., Stephanie Yerdelen, Thomas McGlone, Ikechukwu I. Onyemelukwe, Blair Johnston, Alastair J. Florence, and Nuno M. Reis. 2016. "A Factorial Approach to Understanding the Effect of Inner Geometry of Baffled Meso-scale Tubes on Solids Suspension and Axial Dispersion in Continuous, Oscillatory Liquid-solid Plug Flows". figshare. https://hdl.handle.net/2134/22603. 


\section{Accepted Manuscript}

A factorial approach to understanding the effect of inner geometry of baffled meso-scale tubes on solids suspension and axial dispersion in continuous, oscillatory liquid-solid plug flows

Louisa N. Ejim, Stephanie Yerdelen, Thomas McGlone, Iyke Onyemelukwe, Blair Johnston, Alastair J. Florence, Nuno M. Reis

PII: S1385-8947(16)31248-7

DOI: http://dx.doi.org/10.1016/j.cej.2016.09.013

Reference: CEJ 15723

To appear in:

\section{Chemical Engineering Journal}

Received Date: 18 May 2016

Revised Date: $\quad 2$ September 2016

Accepted Date: $\quad 3$ September 2016

Please cite this article as: L.N. Ejim, S. Yerdelen, T. McGlone, I. Onyemelukwe, B. Johnston, A.J. Florence, N.M. Reis, A factorial approach to understanding the effect of inner geometry of baffled meso-scale tubes on solids suspension and axial dispersion in continuous, oscillatory liquid-solid plug flows, Chemical Engineering Journal (2016), doi: http://dx.doi.org/10.1016/j.cej.2016.09.013

This is a PDF file of an unedited manuscript that has been accepted for publication. As a service to our customers we are providing this early version of the manuscript. The manuscript will undergo copyediting, typesetting, and review of the resulting proof before it is published in its final form. Please note that during the production process errors may be discovered which could affect the content, and all legal disclaimers that apply to the journal pertain. 


\section{A factorial approach to understanding the effect of inner geometry of baffled meso-scale tubes on solids suspension and axial dispersion in continuous, oscillatory liquid-solid plug flows}

Louisa N. Ejim, ${ }^{\mathrm{a}}$ Stephanie Yerdelen, ${ }^{\mathrm{b}}$ Thomas McGlone, ${ }^{\mathrm{b}}$ Iyke Onyemelukwe, ${ }^{\mathrm{a}}$ Blair Johnston, ${ }^{\mathrm{b}}$ Alastair J. Florence, ${ }^{\mathrm{b},{ }^{*}}$ Nuno M. Reis ${ }^{\mathrm{a},{ }^{*}}$

${ }^{a}$ EPSRC Centre for Innovative Manufacturing in Continuous Manufacturing and Crystallisation c/o Department of Chemical Engineering, Loughborough University, Loughborough, Leicestershire, LE11 3TU, UK. E-mail: n.m.reis@lboro.ac.uk. Tel: +44(0)1509222 505; Fax: +44(0)1509223923

${ }^{\mathrm{b}}$ EPSRC Centre for Innovative Manufacturing in Continuous Manufacturing and Crystallisation c/o Strathclyde Institute of Pharmacy and Biomedical Sciences, University of Strathclyde, Technology and Innovation Centre, 99 George Street, Glasgow G1 1RD, UK. Email: alastair.florence@ strath.ac.uk; Tel: +44 (0)141 548 4877; Fax: +44 (0)141 5522562

\footnotetext{
* Corresponding author: n.m.reis@lboro.ac.uk
} 


\section{ABSTRACT}

Oscillatory flow reactors (OFRs) are a new generation of tubular mixing and reaction equipment uniquely capable of combining continuous near plug flow with homogeneous particle suspension, yet the design of OFRs for liquid-solid and multi-phase flow processes relies on rules established during the past two decades from single, liquid-phase studies. A Design of Experiment (DoE) approach was herein implemented for establishing the relationship between four key geometrical parameters of the inner tube baffles and both the suspension of particles and the axial dispersion for liquid-solid continuous flows in $10 \mathrm{~mm}$ internal diameter $(d)$ meso-scale tubes with periodic baffles. The parameters evaluated were the orifice open diameter, $d_{o}=0.35 d-0.50 d$; the open cross section, $\alpha=0.12 d-0.25 d$, constriction spacing, $l=1.5 d-3.0 d$, and baffle shape (sharp vs smooth edged). A total of ten tubes were tested, five consisting of smooth periodic constrictions (SPC) and the other five of sharp edged periodic constrictions (SEPC) according to a complete $2 \times 2$ factorial design with 1 central point. Each tube was experimentally evaluated via optical imaging of suspended monodispersed polyvinyl chloride (PVC) particles. Both SPC and SEPC meso-tubes were capable of delivering a near plug behaviour and the values of axial dispersion coefficient $\left(D_{c}\right)$ estimated for the solids were in the range of $1.0-2.2 \times 10^{-4} \mathrm{~m}^{2} \mathrm{~s}^{-1}$. In contrast, the minimum (critical) fluid oscillation conditions required for full suspension of particles varied significantly, in general with the SPC tubes requiring up to $50 \%$ lower amplitude for full particles suspension. Overall, $\alpha$ revealed the dominant parameter in controlling solids backmixing and, and the inner tube geometry requiring the lowest energy input for homogenous particle suspension and minimum $D_{c}$ (i.e. sharpest residence time distribution) presented a $\| / d=3, d_{o}=0.35 d, \alpha=12 \%$ and SPC design. This study is believed to support the future design of optimised meso-scale OFR systems for continuous screening and manufacturing of value-added liquid-solid and multi-phase systems, such as catalytic and crystallisation processes.

Keywords: Oscillatory flow; meso-scale reactor; smooth periodic constrictions; design of experiment; solid-liquid flow; plug flow 


\section{Introduction}

Oscillatory flow reactors (OFRs) are a new generation of mixing equipment capable of delivering plug flow behaviour decoupled from the net flow [1] for applications in chemical and biological systems. Several key applications have been explored as a result of their unique ability to accommodate slow reactions in a reactor of length to diameter ratio a magnitude lower than conventional tubular plug flow reactors. The plug flow behaviour in OFRs results from effective flow separation around the edge of periodically spaced orifice baffles, which promote the formation of vortices by interacting with the periodic, oscillatory fluid. This yields secondary mixing in the tube, resulting in radial velocities of the same order of magnitude as the input axial velocities [2]. Subjecting OFRs to a net flow with a superimposed reversing oscillatory component of the correct magnitude, leads to efficient controllable fluid mixing, enhanced heat and mass transfer, good particle suspension and reduced shear [3]. The need for small scale continuous manufacturing platforms and scalable continuous screening devices has led to a relatively recent development in OFR technology to down-scaling the reactor to meso-scale [3], which can be broadly defined as internal diameter, $d$ in the range of 1 to $10 \mathrm{~mm}$. Meso-scale OFRs are becoming considerably attractive for continuous catalytic and pharmaceutical manufacturing owing to their small volume and ability to cope with multiphase flows including particles and operate at low flow rates with reduced feed-stock materials and waste. Nonetheless, currently the design of OFRs relies on "rule of thumb" concepts established in the past two or three decades based on single, liquidphase dispersion [4] and mixing [5] studies which are actually paramount to establish the relationship between the inner baffled-tube geometry and solids flow, especially in mesoscale OFRs.

The fluid mechanics in OFRs depend on several key geometrical parameters such as the baffle spacing $(l)$, orifice diameter $\left(d_{0}\right)$ and open cross sectional area $(\alpha)$, however the relationship between these geometrical parameters and continuous particles suspension/flow has not been yet established in literature. Baffle spacing has a significant effect on the behaviour of fluid as it determines the growth extent of generated eddies [5]; the vortices formed are unable to mix across the entire inter-baffle region if $l / d$ is too small. Instead, eddies generated are distorted as a result of interaction with the baffles, therefore restraining the growth of vortices within each inter-baffle region [6]. If $l / d$ is too large, this leads to vortices that do not propagate through the full volume of the inter-baffle region, producing stagnant regions, [2] leading to tailing and significant deviation from ideal plug flow. 
$\mathrm{Ni}$ and Stevenson [6] studied the effect of baffle distance in respect to the fluid mixing; it was observed that increasing the gap size increased the mixing time regardless of the fluid oscillation frequency $(f)$ and centre-to-peak amplitude $\left(x_{0}\right)$. In conventional OFRs, the optimum baffle spacing was previously found to be $l=1.5 d$ for optimum liquid mixing [7]. However, Reis [8] pioneered the scale-down of OFRs and reported unmatched performances on a $4 . .4 \mathrm{~mm}$ i.d. meso-scale OFRs using a baffle spacing of $l / d=3$.

The width of the vortices generated in a baffle cavity and the shape of eddies are also highly influenced by the baffle open area $\alpha=\left(d_{o} / d\right)^{2}$ with larger values giving rise to narrow vortices and hence, poor mixing. Ni et al. [5] reported that $\alpha \sim 0.25$ resulted in the shortest mixing time. According to Baird and Stonestreet [9] a typical value of $\alpha$ is in the range of 0.2-0.4, but Ni et al. [5] reported an optimal value of $\alpha=0.20-0.22$ (see Table 1). An energy dissipation and heat transfer study in OFRs used $\alpha=0.34$ [1] whilst $\alpha=0.21$ was used for a bioreactor application by Gaidhani et al. [10]. Clearly, there appears to be no agreement in literature about the optimum value of $\alpha$ to be used, nevertheless from the fluid mechanics perspective smaller orifice diameter, $d_{o}$ constricts the fluid to a greater extent as it flows through each baffle resulting in wider vortex formation and more effective radial mixing conditions.

Table 1 summarises the optimum geometrical parameters used in the design of conventional bench-scale and pilot-scale OFRs established based on liquid phase mixing studies[11]. The fluid mechanisms in OFRs are governed by a number of dimensionless groups, in particular the oscillatory Reynolds number:

$$
R e_{o}=\frac{2 \pi f x_{o} d \rho_{f}}{\mu}
$$

The Strouhal number described as

$$
S t=\frac{d}{4 \pi x_{o}}
$$

and the net flow Reynolds number:

$$
R e_{n}=\frac{u d \rho_{f}}{\mu}
$$

where $\rho_{f}$ is the fluid density $\left(\mathrm{kg} \mathrm{m}^{-3}\right), \mu$ is the dynamic fluid viscosity ( $\mathrm{Pa} \mathrm{s}$ ), $u=Q / A$ is the superficial net flow velocity through the tube $\left(\mathrm{m} \mathrm{s}^{-1}\right), Q$ is the volumetric net flowrate $\left(\mathrm{m}^{3} \mathrm{~s}^{-}\right.$ ${ }^{1}$ ) and $A$ is the maximum cross-sectional area of the tube $\left(\mathrm{m}^{2}\right)$. The $R e_{o}$ number is based on the maximum (peak) oscillatory fluid velocity, $u(t)_{\max }=2 \pi f x_{o}$ which characterises the intensity of mixing [12]. St number describes the stroke of oscillation in relation to particular tube geometry; when too high eddies propagate into the next baffle cavity, thereby reducing 
the number of potential tank in series. The net flow $R e_{n}$ describes the fluid dynamics of additional net flow within the tube [13].

OFRs can be operated vertically, horizontally or at any angle [14], and there are two different types of mechanisms: $i$ ) pulsing fluid and $i$ ) oscillating baffles. The pulsing fluid is generated either by a diaphragm, pump, piston or bellows, while the oscillating baffle involves the use of mechanical, hydraulic, pneumatic or electro-mechanical devices [14]. Effective mixing can be generated at reduced shear rates as a result of vortices formed as fluid is forced through each periodic constriction. For a continuous flow, a second pump is often required for net flow through the system [2]. Unlike stirred tank reactors and conventional tubular reactors that rely on stirring mechanical and turbulent flow conditions to provide mixing, the OFR uses oscillations to produce eddy vortices. The vortices are formed periodically along the entire length of the tube/reactor enabling each inter-baffle zone to act as a finite number of continuous stirred tank reactors connected in series [2]. A key difference between an OFR and a conventional tubular reactor is the controllable mixing intensity achievable in the former which does not rely on energy intensive turbulent flow conditions. This control is achieved, not by altering the flow rate but by changing the oscillating conditions which impacts the size and frequency of vortex formation [2].

Residence time distribution (RTD) is a major tool for analysis of both kinetic and transport models in continuous flow and reacting systems. It is possible to obtain parameters that characterise the flow dispersion by fitting parameters of a model representing the flow in the reactor. These parameters are often dependent on the fluid properties, flow nature and reactor geometry [15]. Several studies have used RTD to diagnose continuous mixing and plug flow in meso-scale OFRs, however, solid RTDs in OFRs has not yet been reported. Additionally, there is no current literature around the relationship between single-phase RTD and liquid-solid or multi-phase flow RTD. It is therefore paramount to establish: (1) that near plug flow is actually achievable for continuous liquid-solid flow in OFRs; (2) meso-scale OFRs with smooth periodic constrictions (SPCs) [8] favour sharper near-plug flow for liquidsolid flow compared to conventional OFRs design based on sharp constrictions.

A study carried out by Reis et al. [3] involved screening fluid oscillation conditions for full suspension of solid catalysts and optimised mixing in a $4.4 \mathrm{~mm}$ i.d. SPC meso-tube using particle image velocimetry (PIV) and computation fluid dynamics (CFD). In another study [12] on the same OFR geometry it was concluded that an increase in $f$ increases the radial mixing rates. The effect of baffle geometry on RTD of meso-scale OFRs was further studied using three different meso-tube baffle designs with integral, helical and axial circular 
baffles [16]. Phan and Harvey [17] characterised liquid RTD of a meso-scale OFR with helical baffles using the pulse RTD technique using a KCI tracer, and showed that a high degree of mixing was possible using the tanks-in-series model. Similar results were obtained by Zheng and Mackley [18] by investigating the performance of a $\sim 10$-meters long mesoscale OFR with U-bends using the plug flow with axial dispersion model. RTDs in helically coiled and chaotic twisted pipes have been modelled, by comparing mixing in both configurations by means of a two-measurement point technique using the axial dispersion plug flow model [19].

In this study, ten internal tube shapes for $10 \mathrm{~mm}$ i.d. meso-scale OFRs were screened by conducting continuous solid-liquid RTD experiments to characterise the performance of each tube for solid suspension and continuous solid-liquid flow according to a full factorial Design of Experiment (DoE) approach. The methodology aimed to determine the inner mesoscale tube geometry combination that favours both homogeneous particle suspension and sharp RTD for liquid-solid flows, presenting the highest potential for continuous solid-liquid reactions and crystallisation processes. Solid RTD is a crucial aspect in understanding the material flow in many industrial processes such as the continuous crystallisation in pharmaceutical, food, polymers and catalyst products.

\section{Materials and Methods}

\subsection{DoE approach}

A DoE approach, using MKS' MODDE 10.1 (MKS Data Analytics solutions, Sweden) consisting of a complete $2 \times 2$ factorial design with 1 central point was used to investigate the impact of baffle shape (smooth versus sharp edge), $d_{0}, l$ and $\alpha$ for ten meso-tubes (Figure 1) in respect to batch suspension and continuous RTD for liquid-solid flow. The meso-tubes consisted of several geometries with different dimensions however each tube had $d=10 \mathrm{~mm}$ and featured a $1 \mathrm{~m}$ long jacketed glass section. Table 2 presents the targeted dimensions $\left(l, d_{o}\right)$ and the actual dimensions measured after fabrication of the tubes. Some of the tubes were fabricated such that $l$ differed compared to the conventional spacing of $1.5 d$ first reported by Brunold et al. [7]. Four tubes had $l=30 \mathrm{~mm}$ resulting in $l / d=3$ (fully studied for SPC by Reis [8]), two tubes with $l=22.5 \mathrm{~mm}$ were also screened, resulting in a range of $\alpha=0.12$ 0.25 (Table 3). From the total ten tubes screened five tubes presented SPCs, and five sharp edge periodic constrictions (SEPC), in the range of $l / d=1.5-3.0$ (Table 3). Due to differences on the internal shape of the tubes, the internal volume varied between $48-72 \mathrm{~mL}$, which 
results in differences in the mean hydraulic time, $\tau$ which were accounted for in the RTD characterisation.

\subsection{Batch suspension of particles}

Each tube was connected horizontally to a piston-driven electromagnetic oscillator controlled by an amplifier (PA100E, Ling Dynamic systems Ltd, UK) and a signal generator (PO100, Ling Dynamic systems Ltd, UK) providing fluid oscillations in a range of $f$ and $x_{0}$. A micro mesh (The Mesh Company, UK) with a fine wire diameter made of stainless steel fibre, was fitted at this end of the tube to prevent the backflow of particles into the piston as illustrated in Figure 2. Then, a fixed mass $6.8 \mathrm{~g}$ of monodispersed PVC particles (SigmaAldrich, UK) targeting 10 wt.\% for the slurry were homogenously suspended in deionised water at room temperature $\left(20{ }^{\circ} \mathrm{C}\right)$ using fluid oscillation. The PVC particle size distribution (PSD) was determined using the QICPIC analytical system with a RODOS dispersion unit and a $4-3000 \mu \mathrm{m}$ optical lens (Sympatec, NJ, USA). This highlighted the spherical nature of the particles and gave a unimodal, narrow PSD with $D_{50}=141.30 \mu \mathrm{m}$ based on equivalent perimeter of a circle (EQPC). PVC particles in this size range were chosen to represent typical conditions in pharmaceutical crystallisation and their ease to observe optically. Once the particles were homogenously suspended in the tubes, they were allowed to completely settle after which oscillation was reapplied, and $x_{o}$ increased until it was visually detected that all particles were fully suspended. The minimum $x_{o}$ required to fully suspend the PVC particles and herein described as the critical amplitude $\left(x_{o, c r i t}\right)$ was measured from the liquid level of a $0.5 \mathrm{~mm}$ i.d. vertical plastic tube, connected to the other end of the tube (Figure 2). At least three experimental repeats were carried out at values of $f=2,5$ and $7 \mathrm{~Hz}$ where the $x_{o, c r i t}$ varied for each tube depending on its geometry. The efficiency of the radial transport which is paramount to attain homogeneous particle suspension depended on variables such as $f, x_{o}, \alpha$ and baffles thickness.

\subsection{Continuous RTD for liquid-solid flow}

Using the same PVC particles injection technique as described for batch suspension, the homogenously suspended particles were gradually washed out of the tubes using deionised water at a steady net flow. A CCD camera (QICAM 12-bit, Qimaging, UK) located adjacent to the outlet of the reactor (the centre of the interrogation area was at a distance of $0.82 \mathrm{~m}$ from the tube inlet), was used to measure the absorbance of the liquid-solid suspension (which is related to the concentration of particles) during the washout procedure. A light source $(300 \mathrm{~V}, 22 \mathrm{AWG}$, Yue Yang, China) provided illumination of a clear baffle space 
cross section of the reactor tube. This was achievable as a result of rectangular Perspex optical box filled with glycerol (99+\%, Acros organics, UK), fitted around the meso-tube. The glycerol minimized optical distortion with a refractive index of $\sim 1.47$ at $20{ }^{\circ} \mathrm{C}$ [20], similar to Perspex and glass; hence the glass jacket of the tubes was also filled with glycerol. A diffuser was used to spread the light and provide homogeneous illumination of the particles, minimising inaccuracy in particle concentration measurements.

The absorbance of the liquid-particles suspension was calibrated against known PVC particles concentration to ensure accurate concentration measurements of the particles leaving the system. The operating oscillatory conditions $f, x_{o}$ and $u$, were already established before PVC particles injection, the distinct $x_{o, \text { crit }}$ of the tubes were obtained from the batch suspension studies (Figure 3 ) at a fixed $f=5 \mathrm{~Hz}$; it is easier to suspend particles at high $f$ and low $x_{o}$ than at low $f$ and high $x_{o}$ [21]. For effective particle suspension, strong eddy vortices are required to produce a "lifting" force in the particles without compromising the plug flow behaviour. A continuous water flow rate of $17.8 \mathrm{ml} \mathrm{min}^{-1}$ equivalent to $R e_{n} \sim 42$ was used to allow a gradual washout of particles with a mean $\tau=10-15$ min. In a practical situation, several tubes would be stacked in series in order to produce a residence time of up to few hours.

Each tube was first filled with water before injecting $6.8 \mathrm{~g}$ of PVC particles into the oscillating unit using a $25 \mathrm{~mL}$ syringe attached to the inlet, the gentle fluid oscillations and net flow dragged the solid particles into the tube. After homogeneity of particles along the reactor has been established, the wash out was carried out by a pump (Cole Parmer, UK) which provided a net flow whilst the camera recorded particles of an optical density within the interrogation volume. These camera measurements were processed using ImageJ software $(\mathrm{NIH}, \mathrm{USA})$ and the mean pixel grey scale converted to concentration of PVC particles $\left(\mathrm{g} \mathrm{ml}^{-}\right.$ ${ }^{1}$ ) using Beer Lambert's law:

$$
A b s=\varepsilon l_{p} c=-\log _{10} \frac{I_{0}}{I}
$$

where $c$ is the concentration of particles $\left(\mathrm{g} \mathrm{mL}^{-1}\right), l_{p}$ is the light path distance $(\mathrm{m}), \varepsilon$ is the wavelength-dependent molar absorptivity coefficient $\left(\mathrm{M}^{-1} \mathrm{~cm}^{-1}\right), I_{0}$ is the intensity of incident light beam entering the optical box taken as grey scale of baseline and $I$ is the intensity of the light emerging out of the box taken a s grey scale of baseline. The camera calibration returned 
a linear relationship between absorbance and particles concentrations with cross-correlation coefficient, $R=0.998$ for a range of particles concentration of $0-20 \mathrm{wt} \%$.

In each experiment, one frame was captured per second and a total of approximately 600 frames processed per experiment depending on the washout time of each individual meso-tube. Camera recording during the wash out continued until particles were no longer visible within the meso-tube. The time for complete washout varied slightly with each tube geometry, but an average of $10 \mathrm{~min}$ was used. At any time, $t>0$, the fraction of particles remaining in the system was given as:

$$
W(t)=\frac{C_{o u t}(t)}{C_{0}}
$$

where $C_{\text {out }}(t)$ is the outlet concentration of PVC particles at time $t$ and $C_{0}$ is the initial PVC particle concentration. These particles must have entered the tube before time, $t=0$, since no particles were fed after this time, hence the particles have residence times of $t$ or longer. The washout experiment applies to any flow system and is generally preferred since $W(\infty)=0$ will be known but the cumulative normalised particle concentration, $F(\infty)=C_{0}$ (from a step input experiment) must usually be measured [22]. The wash out function is also considered by many designers to be the best function to use for defining the moments of the distribution function [23]. The cumulative distribution function, $F(t)$ is related to the wash out function by:

$$
F(t)=1-W(t)
$$

The mean residence time of particles, $\bar{t}$ was determined by finding the area under the $F(\theta)$ curve:

$$
\bar{t}=\frac{\sum_{i} t F_{i} \Delta t_{i}}{\sum_{i} F_{i} \Delta t_{i}}
$$

The normalised cumulative distribution $F(\theta)$ was plotted based on the dimensionless residence time given as:

$$
\theta=\frac{t}{\bar{t}}
$$

where $t$ is the experimental time. A value of $\bar{t}$ similar to $\tau$ demonstrates that the solid mean residence time is similar to the mean liquid hydraulic time, whereas values of $\bar{t} \gg \tau$ means solids are delayed within the meso-tube. The purpose of creating normalised distribution functions was to enable the direct comparison of flow performance inside the meso-scale OFRs of different sizes. For example, when the normalised function $E(\theta)$ is used, all perfectly 
mixed continuous stirred tank reactors (CSTRs) have numerically the same RTD, but when the same simple function $E(t)$ is used, numerical values of $E(t)$ differ substantially for different CSTRs [24].

\subsection{Determination of axial dispersion coefficient}

The experimental $F(\theta)$ obtained from the RTD studies was fitted to the analytical solution of a plug flow with axial dispersion model with open-open boundaries [25]. The fitting represents a plug flow of fluid on top of which is superimposed some degree of backmixing, the magnitude of which is independent of position within the vessel [25]. The purpose of fitting the experimental results to the axial dispersion model is to determine a realistic flow of fluid in a reactor which often involves backmixing, redistribution of materials either by slippage or eddies as observed during the course of the experiments. In contrast to tanks-in-series, this model is usually capable of accounting for large deviations from plug flow. This effect is quantified by an effective axial dispersion coefficient $D_{c}\left(\mathrm{~m}^{2} \mathrm{~s}^{-1}\right)$ given by a dimensionless axial dispersion parameter $\left(D_{c} / u L\right)$ represented by the following differential equation:

$$
\frac{\partial C}{\partial \theta}=\left(\frac{D_{C}}{u L}\right) \frac{\partial^{2} C}{\partial z^{2}}-\frac{\partial C}{\partial z}
$$

The dimensionless group $D_{c} / u L$ in Equation (9) is often called also the vessel dispersion number [25], a parameter which measures the extent of axial dispersion; $C$ is the solids concentration; $z=x / L$ is dimensionless length; $x$ is the axial position $(\mathrm{m}), u$ is the mean axial velocity $\left(\mathrm{m} \mathrm{s}^{-1}\right)$ and $L$ is the axial position of the test section relative to the tube washout inlet (m). For small deviations from plug flow $\left(D_{c} / u L<0.01\right)$, the open-open boundary condition happens to be the only physical situation where the analytical expression for the $E(\theta)$ curve is straightforward, this is derived as [25]:

$$
E(\theta)=\frac{1}{\sqrt{4 \pi\left(D_{c} / u L\right)}} \exp \left[-\frac{(1-\theta)^{2}}{4 \theta\left(D_{c} / u L\right)}\right]
$$

The plug flow with axial dispersion model was herein used with two fitting parameters, being $t_{m}=u / L$ and $D_{c}$. Since $L$ is constant, $t_{m}$ varies as $u$ is varied. The shape of the $F(\theta)$ curve was obtained by integrating $E(\theta)$ in equation (10): 


$$
F=\int_{0}^{\theta} E_{\theta} d \theta=\int_{0}^{t} E d t
$$

The experimental and model $F(\theta)$ curves were best fitted using Excel's Solver tool giving the best numerical fit of the RTD curves with the model, by a least squares minimisation method. In reality, the boundary of the experimental RTD was a closed-open condition, with a through-the-wall measurement; the mesh restricted the particles from flowing back into the oscillation unit. However, the particles exit region was not constricted and particles able disperse [26]. As analytical solution of Equation (8) with closed-open boundaries is non-trivial, the open-open analytical solution provided by Levenspiel [25] provided a fair approximation for comparison purposes of the different tube geometries.

\section{Results and Discussion}

OFRs have been developed and applied to relevant industrial applications for decades, however there is no record to date in literature of the ability of this new generation of tubular mixing technology to deliver near plug flow for liquid-solid and multiphase flows. Also, it remains unclear the effect of the inner tube geometry on the performance of liquid-solid and multiphase OFRs. To address these major knowledge gaps experiments were carried out on both batch and continuous flow configurations using model monodispersed PVC particles believed to mimic the behaviour of micron-sized crystal particles which is relevant for industries such as pharmaceuticals and food. A DoE screening involved manufacturing and testing five SPC tubes of distinct geometries (in the range of $l / d=1.5-3$ ) and an additional five SEPCs tubes in the same range. Both the SPCs and SEPC tubes were compared in terms of their axial dispersion coefficients, mean experimental and modelled residence time and the minimum conditions for effective particle suspension. Seven model terms, including factors $\alpha$, $l / d$ and constriction type (sharp vs smooth) and their interaction terms, were fit with DoE software to experimental responses from batch particle suspension and continuous RTD data,

and returned the following coefficients: $R^{2}=0.72$ for $x_{o, \text { crit }}$ at $f=2 \mathrm{~Hz} ; R^{2}=0.79$ for $x_{o, \text { crit }}$ at $f$ $=5 \mathrm{~Hz} ; R^{2}=0.73$ for $D_{c} / u L, R^{2}=0.91$ for $\tau ; R^{2}=0.88$ for $D_{c}$; and $R^{2}=0.96$ for $\bar{t}$. This is discussed in detail in sections 3.1 and 3.2.

\subsection{Effect of inner meso-tube geometry on batch suspension of particles}

The effect of meso-tube geometry on critical fluid amplitude for full suspension of particles, $x_{o, c r i t}$ was carried out in batch mode at $20{ }^{\circ} \mathrm{C}$ at three values of $f=2,5$ and $7 \mathrm{~Hz}$. The 
value of $x_{o, c r i t}$ provides information on the minimum fluid amplitude to fully suspend PVC particles (see Figure 3), therefore being an indirect quantitation of the extent of radial mixing generated by each particular tube geometry. Homogenous particle suspension was easier to obtain with tubes placed at a horizontal position rather than at an angle or vertical position [3] and it also offers a more compact design, therefore the tubes were tested in full horizontal position. The different geometries tested required very distinct values of $x_{0}$ to fully suspend the PVC particles (Figure 4). As expected, the value of $f$ had an impact on the particles suspension, as the particles were easily suspended at a reduced $x_{0}$ when $f$ was increased from 2 to $7 \mathrm{~Hz}$. Figure 4a shows the meso-scale tubes with SPC geometries in general required lower $x_{o, \text { crit }}$ compared to the SEPC of the same $l$ and $d_{o}$. For example SPC tube $B$ and SEPC tube $F$ with same $l=15 \mathrm{~mm}$ and $d_{o}=5 \mathrm{~mm}$ at a fixed $f=2 \mathrm{~Hz}$, presented a value for $x_{o, c r i t}$ of $6.7 \mathrm{~mm}$ and $9.8 \mathrm{~mm}$, respectively. This is believed to be linked to the dead corners of the SEPC tubes requiring higher $x_{o}$ to fully suspend particles.

In general, within the SPC geometries, $x_{o, c r i t}$ was smaller for geometries with larger $l$ and smaller $d_{o}$. For instance, tubes $A\left(l=30 \mathrm{~mm}, d_{o}=3.5 \mathrm{~mm}\right), C\left(l=22.5 \mathrm{~mm}, d_{o}=4.2 \mathrm{~mm}\right)$ and $D\left(l=15 \mathrm{~mm}, d_{o}=3.5 \mathrm{~mm}\right)$ had the lowest $x_{o, \text { crit }}$ (see Figure $4 \mathrm{a}$ ). Tube A required overall the lowest value of $x_{o}$ for full PVC particles suspension at these conditions; it is also a linear scale up of the SPC developed by Reis et al. [27] with $l=13 \mathrm{~mm}, d_{o}=4.5 \mathrm{~mm}$, but the same aspect ratios $l / d=3$ and $\alpha=12 \%$. The significant differences in particle suspension can be explained by differences in power input/dissipation and extent of radial mixing.

The efficiency of particle suspension by $f$ and $x_{o}$ can be linked to the settling velocity of the particles. The fluid velocity (in this case radial as the tubes were placed horizontally) must be similar to the particles terminal velocity to achieve suspension in order for the particles to be lifted up and over the baffled tube wall. The dominance of the radial velocity during the oscillatory flow minimizes the particle settling velocity; the periodic upward motion increases the fluid drag force and helps to suspend the particles in liquids. The maximum steady-state settling velocity of the particles $u_{p}$ can be estimated from Stokes' law:

$$
u_{p}=\sqrt{\frac{4}{3} \frac{1}{C_{D}} g d_{p}\left(\frac{\rho_{p}-\rho_{f}}{\rho_{p}}\right)}
$$

where $\rho_{p}$ is the density of the particles, $\rho_{f}$ is the density of water, $g$ is the acceleration due to gravity $\left(\mathrm{m} \mathrm{s}^{-2}\right), d_{p}$ is the diameter of the particles and $C_{D}$ is the drag coefficient, which has a value of 2.1 [13] at $R e_{n}=42$. The particles are assumed to be spherical as suggested by the 
PSD measurement shown in Figure 5 and the calculated particle settling velocity distribution using Equation (12) is also presented. The volume-weighted mean $u_{m}$ was calculated as 0.048 $\mathrm{m} \mathrm{s}^{-1}$ for the size ranges given above. This was computed by taking the average of all $u_{p}$ values within the given particle size range. Because of the monodispersed nature of the particles, a higher particles density might have a higher terminal velocity thus requiring a higher $f$ and $x_{o}$ [28]. Oscillatory flow in a baffled tube is typically able to suspend solid particles up to $20 \mathrm{wt} . \%$ [28]; in this study $10 \mathrm{wt} . \%$ of PVC particles were used.

The peak oscillatory axial velocity, $u(t)_{\max }=2 \pi f x_{o}$ required for complete suspension of the particles (Figure 4b) should be in the order of magnitude of $u_{m}$ in order to achieve a high energetic efficiency of particle suspension for the reactor (i.e. $u(t)_{\max }>>u_{m}$ ). For all 10 tubes the value of $u(t)_{\max }$ calculated ranged from $0.021-0.124 \mathrm{~m} \mathrm{~s}^{-1}$, with tube $I$ (SEPC, $l / d=$ 3) and $E(\mathrm{SPC}, l / d=3)$ having the largest values of 0.089 and $0.069 \mathrm{~m} \mathrm{~s}^{-1}$, respectively (Figure $4 \mathrm{~b}$ ) at $5 \mathrm{~Hz}$, resulting in values of $u(t)_{\max }$ up to 2.6-fold greater than the mean $u_{m}$. Due to differences in inner shape of the tubes, the ratio between the input axial velocities and the generated radial velocities will naturally vary, and a lower $u(t)_{\max }$ value is an indicator that a particular geometry is more effective in generating the relevant extend of radial mixing required for full suspension the particles.

The differences in batch suspension performance of the SPCs and SEPCs can also be explained with the power dissipation of the tubes, which is linked to differences in spatial arrangement of the constrictions. The power density, $P / V\left(\mathrm{~W} \mathrm{~m}^{-3}\right)$ provides an understanding of the baffle geometries based on the fluid oscillation requirement per unit volume for each meso-scale tube. In this study, the quasi-steady flow model was used as it applies to conditions of high $x_{o}$ and low $f$. The quasi-steady flow model was first derived for packed bed columns and subsequently used for pulsed columns [29] as:

$$
\frac{P}{V}=\frac{2 \rho_{m} N}{3 \pi C_{d}^{2}} \frac{1-\alpha^{2}}{\alpha^{2}} x_{o}^{3} w^{3}
$$

where $N$ is the number of baffles per unit length, $w=2 \pi f$ is the angular frequency, $\rho_{m}$ is the density of PVC-water mixture and $C_{d}$ is the orifice discharge coefficient which is usually 0.7 [30]. For high $x_{o}$, the effect of the $x_{o, c r i t}$ on the $P / V$ is plotted in Figure 6a for SPC and SEPC tubes. As predicted from Equation (13), $P / V$ increased with increasing $f$ and $x_{0}$ but being also very sensitive on both $\alpha$ and $l$. Nevertheless, no clear correlation between the $x_{o, c r i t}$ and $\alpha$ was observed (Figure 6b). The SEPC tubes were shown to require higher $P / V$ values compared to 
SPCs in order to achieve comparable particle suspension at a given $f$ and $x_{o}$. Overall at $5 \mathrm{~Hz}$, tube $G$ (sharp, $l / d=3$ ) had the lowest $\alpha=0.12$ and tube $F$ (sharp, $l / d=1.5$ ) had the highest $\alpha$ $=0.25$ resulting in a $P / V$ value of $3.1 \mathrm{kWm}^{-3}$ and $0.8 \mathrm{kWm}^{-3}$ respectively, corresponding to over a 15 -fold range in $P / V$ values.

Amongst all the SPC tubes, tube $B(l / d=1.5)$ had the lowed estimated $P / V$ value of 0.3 $\mathrm{kWm}^{-3}$ with $\alpha=0.25$ at $f=2 \mathrm{~Hz}$. For small values of $\alpha$ the term $\left(1-\alpha^{2} / \alpha^{2}\right)$ increases and Equation (13) predicts a higher extent of intensity [8]. This could be as a result of higher power input requirement of increased mixing efficiency due to the higher dispersion rate. This also suggests the existence of a margin in the uniformity of mixing [5].

Overall, the SPC tubes proved to suspend particles more efficiently than the SEPC tubes with the same dimensions, and SPC tube $A\left(l=30 \mathrm{~mm}, d_{o}=3.5 \mathrm{~mm}\right)$ emerged the most suitable for batch PVC particles suspension since it required the lowest $x_{o}$ for any given $f$ input. Tube A also showed a reduced power density with $P / V=0.4 \mathrm{kWm}^{-3}$ at $5 \mathrm{~Hz}$. Additionally, increasing the oscillation $f$ reduced the particle settling velocity, resulting in reduced $x_{o}$ required for suspension.

\subsection{Effect of meso-tube geometry on continuous RTD of solid-liquid flow}

Solid-liquid RTD technique was used to ascertain the capability of delivering near plug behaviour for continuous liquid-solid flow in the meso-tubes, a relevant engineering aspect not previously demonstrated in OFRs. The particle-liquid flow in the tubes was characterised using the washout RTD method yielding $F(\theta)$ curves corresponding to cumulative PVC particles concentration distribution in the tube. The shape of the $F(\theta)$-curve varies according to the flow behaviour; for near plug flow sharper $F(\theta)$ was expected as presented in Figure 7a for SPC tubes and Figure 7b for SEPC tubes. Although all $F(\theta)$-curves appeared similar in shape as a result of the similarity in the geometries, the SPC tubes showed sharper response as unity was reached at $\theta \sim 1.8$ compared to $\theta \sim 2.0$ for the SEPC tubes. The washout method ensured that the PVC particle concentration was first detected at the maximum before a net water flow rate of $17.8 \mathrm{~mL} \mathrm{~min}^{-1}$ decreased the concentration of particles in the tube with time. Note that the variation in the volume of the tubes (Table 2) means that particle loading should have been adjusted to maintain a constant concentration, however RTD measurements carried out at different solids concentrations (9.4-14.0 wt.\%) fully overlapped (data not shown).

Although sharp edged baffles generated strong eddy vortices, the SPC tubes displayed a more uniform mixing and less extent of backmixing. Overall, both the SPC and SEPC tubes 
provided well-defined and more symmetrical $F(\theta)$-curves as expected for a plug flow with the applied oscillation conditions. To further distinguish the fundamental characteristics of continuous liquid-solid flow in the SPC and SEPC tubes, the experimental mean residence times $(\bar{t})$ and axial dispersion model residence time $\left(t_{m}\right)$ were compared to $\tau$. All experiments were carried out in continuous mode, as it has been previously shown that a small net flow does not affect the extent of oscillatory flow mixing [31].

Experimental optical observations revealed eddy vortices formation during the RTD experiments similar to those showed in Figure 3, thus the solid-liquid flow was modelled as a plug flow with axial dispersion. The extent of deviation from plug flow is characterised by the dimensionless axial dispersion coefficient, $D_{c} / u L$, with values smaller than 0.01-0.02 being considered a perfect plug flow. In this particular study the value of $L$ used was $0.82 \mathrm{~m}$ (with total tube length of $1.0 \mathrm{~m}$ ), therefore $D_{c} / u L$ values calculated will be larger than the ones obtained in a manufacturing unit composed of a number of meso-tubes arranged in series.

The effect of baffle type on axial dispersion was distinguished in the SPC and SEPC baffles by fitting the model with two parameters, $t_{m}$ and $D_{c}$. To estimate $D_{c}$ the model was fitted to the experimental $F(\theta)$-curve which produced values of $D_{c} / u L$ in the range of 0.04 0.08 (Figure $8 \mathrm{a}$ ) for all tubes tested; this means a perfect plug flow can be achieved in a 2-8 meters long meso-scale OFR. Tubes $A$ (SPC), $F$ (SEPC) and I (SEPC) showed the smallest $D_{c} / u L$ values, these tubes present high $\alpha$, and there was no clear trend of dependency of the $D_{c} / u L$ on the baffle type (Figure 8a). Similarly, the values of $D_{c}$ obtained were in the range of $1.0-2.2 \times 10^{-4} \mathrm{~m}^{2} \mathrm{~s}^{-1}$ (Figure 8b), i.e. varying only by 2-fold. Reis et al. [27] reported maximum values of $D_{c}$ in the range of $6.0 \times 10^{-5}-3.4 \times 10^{-4} \mathrm{~m}^{2} \mathrm{~s}^{-1}$ for continuous liquid phase RTD in $4.4 \mathrm{~mm}$ i.d. meso-tube with ratio $l / d=3$, and fluid oscillations of $x_{o}=0-3 \mathrm{~mm}$ and $f$ $=0-20 \mathrm{~Hz}$. This is in accordance with the current experimental results, confirming for the first time that axial dispersion of solid-liquid flow in meso-scale OFRs is comparable to axial dispersion in liquid phase flow. Neverthless, values of $t_{m}$ in Figure $8 \mathrm{c}$ clearly showed that some inner tube geometries (e.g. A and G) are particularly good at trapping solids, with the numerical fitting model predicting a value of $t_{m}$ significantly larger than $\tau$. The consequences of this are discussed in more detail in this section.

The sum of the relative deviation between the calculated (best fit) and experimental $F(\theta)$-curve (by least squares minimisation method) was in the range of 0.13-0.82. Buratti [32] defined the experimental error as the difference between the experimental RTD and the best 
fit with the plug flow with axial dispersion model to be $\sim 13 \%$ (i.e. 0.13 ). With this criteria, tube $C$ (SPC, $l=22.5 \mathrm{~mm}, d_{o}=4.2 \mathrm{~mm}$ ) had the lowest fitting error of 0.13 , followed by tube $F\left(\mathrm{SEPC}, l=15 \mathrm{~mm}, d_{o}=5 \mathrm{~mm}\right)$ and $J\left(\mathrm{SEPC}, l=22.5 \mathrm{~mm}, d_{o}=4.2 \mathrm{~mm}\right)$ with 0.24 error each.

The value of $l / d=3$ for tube $I$ could indicate an optimal baffle spacing $(l=30 \mathrm{~mm}$, SPC) for reduced axial dispersion, since $l$ affects the shape and spread of eddies within each baffle cavity for a given $x_{o}$. This observation is supported by DoE plots (confidence of $95 \%$ ), Figures $9 \mathrm{a}$ and $9 \mathrm{~b}$ show the combined effect of $\alpha$ and $l / d$ on both $D_{c}$ and $D_{c} / u L$, respectively. For the SPC geometries, $D_{c}$ positively correlated with $l / d$ but less on $\alpha$ particularly for lower $D_{c}$ values (Figure 9a). For the SEPC geometries, $\alpha$ had the greater effect on the $D_{c}$; increasing $\alpha$ decreased $D_{c}$. In addition, $l / d$ did not play much of a role until smaller values of $D_{c}$ were observed at larger $\alpha$ values. Interestingly, the normalised $D_{c} / u L$ values showed contrasting effect of $l / d$ and $\alpha$ in the two baffle types tested (Figure 9b); in the SPC tubes, $D_{c} / u L$ was minimised at the lowest values of $\alpha=0.12$ and highest $l / d=3$ tested, whereas in the SEPC reduced solids backmixing was obtained at higher value of $\alpha=0.25$ and lower $l / d=1$ tested. The DoE also highlights both $\alpha$ and shape of constriction as the two largest effectors of $D_{c} / u L$. Table 4 displays the model forms of the response variables $\left(D_{c}\right.$ and $\left.D_{c} / u L\right)$ and independent variables $(l / d$ and $\alpha)$ for the DoE surface plots given in Figures 9a and 9b. Model parameters, standard errors, $p$-values (95\% confidence) and confidence intervals are further detailed in Table 5 for all responses herein modelled. The $p$-value (for a confidence of 95\%) indicate the significance of the parameters; a lowest the $p$-value the higher the statistical significance of that particular parameter.

According to Reis [8], the optimal $l$ should ensure a full expansion of the vortex rings to enable the vortices spread effectively throughout the entire inter-baffle zone whilst minimising the extent of axial dispersion along the tube; a small $l$ value restrains the growth of the yortices and reduces the radial motion within each baffled cell, whereas at large values of $l$ vortices formed behind the constrictions cannot effectively cover the entire interbaffle region. This implies that vortex rings generated are not independent from $l$ as seen in Figure $9 \mathrm{~b}$, and that the shape of the tube constrictions in particular the value of $\alpha$ plays a major role in the overall solids flow characteristics in OFRs.

The $\bar{t}$ values for the solids differed from $\tau$ significantly as shown in Figure 10a, and this discrepancy was consistent for both the SPC and SEPC tubes. The values obtained experimentally were higher than $\tau$, implying that the particles spent more time in the tubes 
than the liquid phase. Similar findings were reported by Phan et al.[17]. The percentage difference was in the range $17-52 \%$ for all 10 meso-scale tubes screened in this work. Surprisingly, additional DoE surface plot representing the ratio $\bar{t} / \tau$ (Figure 10c) showed the increase in residence time of the solids is only due to a reduction on $\alpha$ and independent of the baffle spacing. The models of the response variable $(\bar{t} / \tau)$ and independent variables $(l / d$ and $\alpha$ ) for the DoE plot in Figure 10c are given in Table 4, and the statistical significance of each parameter further detailed in Table 5. Although reduced $\alpha$ values minimised axial dispersion in Figure 9, the strong eddy vortices generated at lower $\alpha$ values resulted in solids trapping and therefore extended residence time of the solids in the meso-tubes. This is the first time to our knowledge such effect is reported with particles, being very relevant for the design of multiphase OFRs. This is presumably linked to centrifugal effect of eddy vortices; reduced values of $\alpha$ leading to higher acceleration of fluid as being pushed through the narrow constrictions, therefore stronger eddy vortices with higher vorticity values. Pereira et al. [33] reported a similar effect on bubbles in a gas-liquid OFR.

The best-fitted mean residence time of the plug flow with axial dispersion model, $t_{m}$ was found to agree closely with values of experimental $\bar{t}$, although $t_{m}$ was in general lower (Figure 10b). Tube $I\left(\bar{t}=248 \mathrm{~s}, t_{m}=239 \mathrm{~s}\right), A\left(\bar{t}=301 \mathrm{~s}, t_{m}=284 \mathrm{~s}\right)$ and $B\left(\bar{t}=209 \mathrm{~s}, t_{m}=197\right.$ s) presented the lowest discrepancy with an error of 4, 6 and $6 \%$ respectively, suggesting these tube geometries produced particles flow characteristic closer to the theoretical plug flow with axial dispersion model described by Equation (9).

Although the optimised geometrical parameters were not obtained from the DoE response plots shown in Figures 9 and 10 collected through a complete factorial DoE approach, those surface plots enabled quantitation of the relevance of each parameter tested. It was observed that the optimum geometry deduced from the plots coincided with a particular inner tube geometry tested in the experiments, for that reason the inner meso-tube geometry was not further optimised.

\section{Conclusions}

This study showed the inner geometry of $10 \mathrm{~mm}$ i.d. meso-tubes and in particular the shape and inner diameter of constrictions strongly dictates the particle suspension capabilities and continuous RTD performance of liquid-solid flows. The batch suspension of particles improved by increasing $f$ and/or $x_{0}$ but depending on the geometry of the tubes some particular inner baffle geometries were more effective in generating significant radial mixing 
at lower oscillatory peak axial velocities of the liquid. Overall, SPCs presented the lowest $x_{0, \text { crit }}$ requirement for full batch suspension of $10 \mathrm{wt} \%$ of PVC monodispersed particles, presumably as a result the presence of dead corners around the baffle region in SEPCs. Power dissipation calculations in the two baffle types also showed that the SEPCs required higher power input, however it also depended on $\alpha$. The solid RTDs in the SPC and SEPC mesotubes were successfully modelled using a plug flow with axial dispersion model, the results produced sharp cumulative distribution curves for all tubes screened. The axial dispersion model fitted well all experimental RTD curves and returned $D_{c}$ values in the same order of magnitude of single-phase, liquid RTD in meso-tubes previously reported in literature. Nevertheless, the open baffle area $\alpha$ identified as the dominant design parameter in controlling solids backmixing and batch suspension of particles, with small values of $\alpha=$ 0.12 resulting in minimised axial dispersion, resulted in extended mean residence times of the particles in the meso-tubes. This was linked to the strong eddy vortices generated at lower values of $\alpha$, that presumably led to trapping of particles, an effect not previously observed in OFRs. Overall, a SPC geometry with $l=30 \mathrm{~mm}, d_{o}=3.5 \mathrm{~mm}, l / d=3$ and $\alpha=0.12$ showed clear flow characteristic advantages over the other meso-tubes studied. The new results and conclusions herein presented will support the design of optimised meso-scale OFR systems for continuous crystallisation and catalytic processes.

\section{Acknowledgements}

This work was supported under EPSRC awards EP/I033459/1 (Centre for Innovative Manufacturing in Continuous Manufacturing and Crystallisation) and EP/K503289/1 (Doctoral Training Centre in Continuous Manufacturing \& Crystallisation. These are in association with a UKRPIF (UK Research Partnership Institute Fund) capital award from the Higher Education Funding Council for England (HEFCE).

\section{Notation}
$A$ cross sectional area of tube $\left(\mathrm{m}^{2}\right)$
$C$ concentration of particles $\left(\mathrm{g} \mathrm{mL}^{-1}\right)$
$C_{d}$ discharge coefficient (dimensionless)
$C_{D} \quad$ drag coefficient (dimensionless)
$C_{o}$ initial concentration of PVC particles $\left(\mathrm{g} \mathrm{mL}^{-1}\right)$ 
$C_{\text {out }}(t) \quad$ outlet concentration of PVC at time $t\left(\mathrm{~g} \mathrm{~mL}^{-1}\right)$

$d$ inner tube diameter (m)

$D_{c}$ axial dispersion coefficient $(\mathrm{m} / \mathrm{s})$

$d_{o}$ inner orifice diameter $(\mathrm{m})$

$d_{p}$ diameter of PVC particles (m)

$E(t)$ exit age distribution function

$E(\boldsymbol{\theta})$ normalised exit age distribution function

$f$ oscillation frequency $(\mathrm{Hz})$

$g$ acceleration of gravity $\left(\mathrm{m} \mathrm{s}^{-2}\right)$

$F(\theta)$ normalised RTD cumulative distribution function

$F(\infty)$ cumulative normalised particle concentration

$I$ intensity of the light emerging out of the box

$I_{0}$ intensity of incident light beam entering the optical box

$l$ baffle spacing $(\mathrm{m})$

$l / d$ length to diameter ratio

$L$ axial position of the test section relative to the tube washout inlet (m)

$l_{p}$ light path distance $(\mathrm{m})$

$N$ number of baffles (dimensionless)

$P / V$ power density $\left(\mathrm{W} \mathrm{m}^{-3}\right)$

$Q$ volumetric flowrate $\left(\mathrm{m}^{3} \mathrm{~s}^{-1}\right)$

$R$ cross-correlation coefficient

$t$ time (s)

$t_{m} \quad$ axial dispersion model mean residence time (s)

$\bar{t}$ experimental mean residence time (s)

$u$ mean superficial flow velocity $\left(\mathrm{mL} \mathrm{min}^{-1}\right)$

$u_{m}$ volume-weighted mean settling velocity of particles $\left(\mathrm{m} \mathrm{s}^{-1}\right)$

$u_{p}$ maximum steady state settling velocity of particles $\left(\mathrm{m} \mathrm{s}^{-1}\right)$

$u(t)_{\max }$ maximum oscillation fluid velocity $\left(\mathrm{m} \mathrm{s}^{-1}\right)$

$V$ inner volume of the tube $\left(\mathrm{m}^{3}\right)$

$v$ kinematic viscosity of the fluid $\left(\mathrm{m}^{2} \mathrm{~s}^{-1}\right)$

$W(t) \quad$ RTD wash out function

$W(\infty) \quad$ cumulative fraction of particles

$x \quad$ axial position $(\mathrm{m})$ 


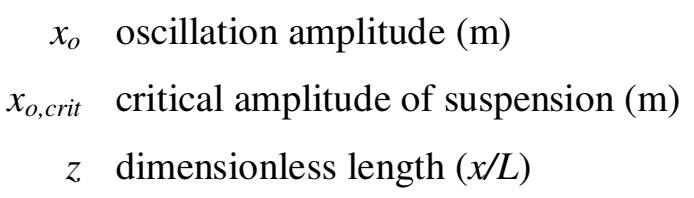

Dimensionless groups

$D$ dimensionless axial dispersion number, $D=D_{c} / u L$

$R e_{n}$ net Reynolds number

$R e_{o}$ oscillatory Reynolds number

St Strouhal number

\section{Greek Letters}

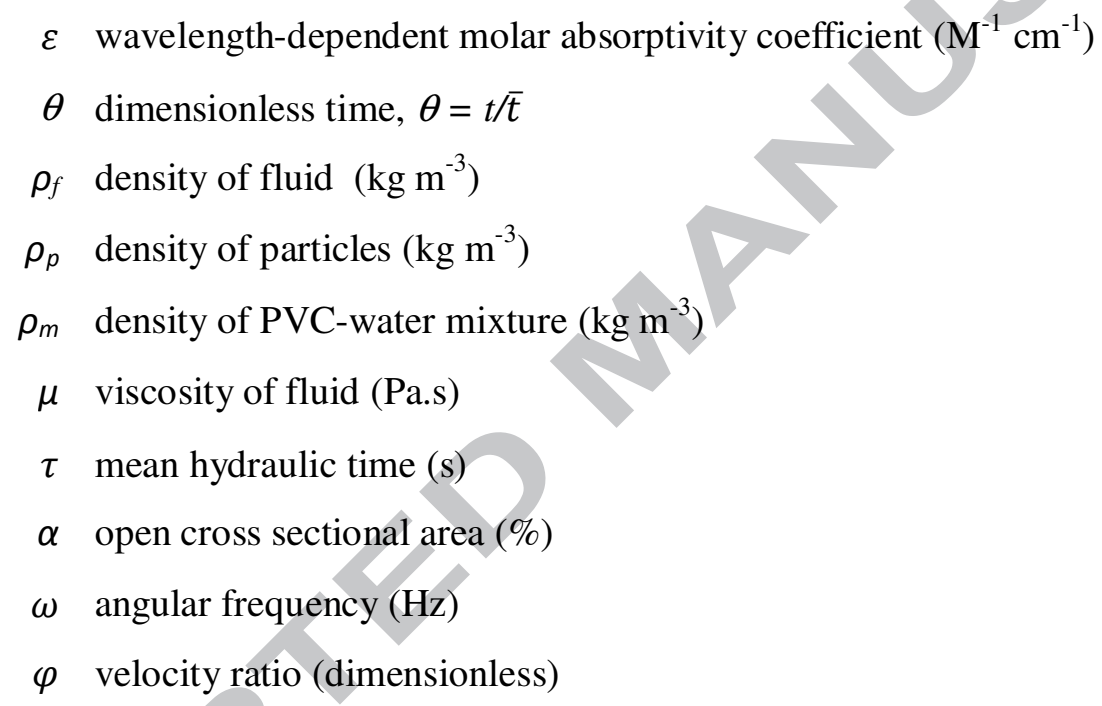

\section{References}

[1] M.R. Mackley, P. Stonestreet, Heat-Transfer And Associated Energy-Dissipation For Oscillatory Flow In Baffled Tubes, Chem. Eng. Sci. 50 (1995) 2211-2224.

[2] M.S.R. Abbott, a. P. Harvey, G. V. Perez, M.K. Theodorou, Biological processing in oscillatory baffled reactors: operation, advantages and potential, Interface Focus. 3 (2012) 20120036-20120036. doi:10.1098/rsfs.2012.0036.

[3] N. Reis, A.P. Harvey, M.R. Mackley, A.A. Vicente, J.A. Teixeira, Fluid Mechanics and Design Aspects of a Novel Oscillatory Flow Screening Mesoreactor, Chem. Eng. Res. Des. 83 (2005) 357-371. doi:http://dx.doi.org/10.1205/cherd.03401. 
[4] E. Lobry, T. Lasuye, C. Gourdon, C. Xuereb, Liquid-liquid dispersion in a continuous oscillatory baffled reactor - Application to suspension polymerization, Chem. Eng. J. 259 (2015) 505-518. doi:10.1016/j.cej.2014.08.014.

[5] X. Ni, G. Brogan, A. Struthers, D.C. Bennett, S.F. Wilson, A systematic study of the effect of geometrical parameters on mixing time in oscillatory baffled columns, Chem. Eng. Res. Des. 76 (1998) 635-642. doi:10.1205/026387698525162.

[6] X. Ni, C.C. Stevenson, On the effect of gap size between baffle outer diameter and tube inner diameter on the mixing characteristics in an oscillatory-baffled column, J. Chem. Technol. Biotechnol. 74 (1999) 587-593. doi:10.1002/(SICI)10974660(199906)74:6<587::AID-JCTB87>3.0.CO;2-C.

[7] C.R. Brunold, J.C.B. Hunns, M.R. Mackley, J.W. Thompson, Experimental observations on flow patterns and energy losses for oscillatory flow in ducts containing sharp edges, Chem. Eng. Sci. 44 (1989) 1227-1244. doi:10.1016/00092509(89)87022-8.

[8] N. Reis, Novel oscillatory flow reactors for biotechnological applications, (2006).

[9] M.H.I. Baird, P. Stonestreet, Energy Dissipation In Oscillatory Flow Within A Baffled Tube, Chem. Eng. Res. Des. 73 (1995) 503-511.

[10] H.K. Gaidhani, B. McNeil, X.W. Ni, Production of pullulan using an oscillatory baffled bioreactor, in: J. Chem. Technol. Biotechnol., 2003: pp. 260-264. doi:10.1002/jctb.766.

[11] T. McGlone, N.E.B. Briggs, C.A. Clark, C.J. Brown, J. Sefcik, A.J. Florence, Oscillatory Flow Reactors (OFRs) for Continuous Manufacturing and Crystallization, Org. Process Res. Dev. 19 (2015) 1186-1202. doi:10.1021/acs.oprd.5b00225.

[12] N. Reis, a. a. Vicente, J. a. Teixeira, M.R. Mackley, Residence times and mixing of a novel continuous oscillatory flow screening reactor, Chem. Eng. Sci. 59 (2004) 49674974. doi:10.1016/j.ces.2004.09.013.

[13] R.B. Bird, W.E. Stewart, E.N. Lightfoot, Transport Phenomena, J. Wiley. (2007) 905.

[14] M. Rasdi, F.R. Binti, Continuous screening using mesoscale oscillatory baffled reactors, (2010).

[15] M. Palma, R. Giudici, Analysis of axial dispersion in an oscillatory-flow continuous reactor, Chem. Eng. J. 94 (2003) 189-198. doi:10.1016/S1385-8947(03)00057-3.

[16] A.N. Phan, A. Harvey, Effect of baffle geometry on residence time distribution of mesoscale oscillatory baffled reactors, (n.d.). 
[17] A.N. Phan, A. Harvey, Development and evaluation of novel designs of continuous mesoscale oscillatory baffled reactors, Chem. Eng. J. 159 (2010) 212-219. doi:10.1016/j.cej.2010.02.059.

[18] M. Zheng, M. Mackley, The axial dispersion performance of an oscillatory flow mesoreactor with relevance to continuous flow operation, Chem. Eng. Sci. 63 (2008) 17881799. doi:10.1016/j.ces.2007.12.020.

[19] C. Castelain, a. Mokrani, P. Legentilhomme, H. Peerhossaini, Residence time distribution in twisted pipe flows: helically coiled system and chaotic system, Exp. Fluids. 22 (1997) 359-368. doi:10.1007/s003480050061.

[20] L.F. Hoyt, New table of the refractive index of pure glycerol at 20 C, Ind. Eng. Chem. 26 (1934) 329-332.

[21] A. Harvey, M.R. Mackley, N. Reis, J.A. Teixeira, A.A. Vicente, Fluid mixing and particle suspension in a novel microreactor, in: 30th Conf. SSCHE, Tatranské Matliare, Slovakia, Citeseer, 2003.

[22] E.B. Nauman, Chemical reactor design, optimization, and scaleup, John Wiley \& Sons, 2008.

[23] R.E. Hayes, J.P. Mmbaga, Introduction to Chemical Reactor Analysis, CRC Press, 2012.

[24] H. Scott Fogler, Elements of chemical reaction engineering, Chem. Eng. Sci. 42 (1987) 2493. doi:10.1016/0009-2509(87)80130-6.

[25] O. Levenspiel, Chemical Reaction Engineering, Ind. Eng. Chem. Res. 38 (1999) 41404143.

[26] C.-Y. Wen, L. Fan, Models for flow systems and chemical reactors, M. Dekker, 1975.

[27] N. Reis, a. a. Vicente, J. a. Teixeira, Liquid backmixing in oscillatory flow through a periodically constricted meso-tube, Chem. Eng. Process. Process Intensif. 49 (2010) 793-803. doi:10.1016/j.cep.2010.01.014.

[28] M.S. Takriff, W. Ramli, W. Daud, Z. Fisal, Z. Alam, Mutiphase oscillatory flow in a baffled tube, 9 (2000) 75-84.

[29] M.H.I. Baird, J.H. Garstang, Power consumption and gas hold-up in a pulsed column, Chem. Eng. Sci. 22 (1967) 1663-1673.

[30] X. Ni, Y. Zhang, I. Mustafa, An investigation of droplet size and size distribution in methylmethacrylate suspensions in a batch oscillatory-baffled reactor, Chem. Eng. Sci. 53 (1998) 2903-2919. 
[31] P. Stonestreet, A.P. Harvey, A Mixing-Based Design Methodology for Continuous Oscillatory Flow Reactors, Chem. Eng. Res. Des. 80 (2002) 31-44. doi:10.1205/026387602753393204.

[32] M.-F. Buratti, Etude des phénomènes de mélange axial dans les colonnes pulsées équipées de garnissage disques-couronnes, (1988).

[33] F.M. Pereira, D.Z. Sousa, M.M. Alves, M.R. Mackley, N.M. Reis, CO2 dissolution and design aspects of a multiorifice oscillatory baffled column, Ind. Eng. Chem. Res. 53 (2014) 17303-17316. doi:10.1021/ie403348g. 


\section{List of Tables}

Table 1. Geometric parameters currently used for designing OFRs

\begin{tabular}{cccc}
\hline Parameter & Symbol & Optimal value & References \\
\hline Baffle spacing & $l$ & $1.5 d$ & {$[6]$} \\
Baffle open area & $\alpha$ & $20-22 \%$ & {$[5]$} \\
Orifice diameter & $d_{o}$ & $0.45-0.50 d$ & {$[5]$} \\
Tube diameter & $d$ & Usually $10-150 \mathrm{~mm}$ & {$[2]$} \\
\hline
\end{tabular}

Table 2. Inner dimensions of meso-tube dimensions used in the DoE

\begin{tabular}{cccccc}
\hline \multirow{2}{*}{$\begin{array}{c}\text { Tube } \\
\text { reference }\end{array}$} & \multicolumn{2}{c}{ Targeted } & \multicolumn{2}{c}{ Measured } & \multirow{2}{*}{ Baffle } \\
& \multicolumn{1}{c}{$\boldsymbol{l}$} & $\boldsymbol{d}$ & $\boldsymbol{l}$ & $\boldsymbol{d}_{\boldsymbol{o}}$ & type \\
& $(\mathbf{m m})$ & $(\mathbf{m m})$ & $(\mathbf{m m})$ & $(\mathbf{m m})$ & \\
\hline A & 30.0 & 3.5 & 29.7 & 3.1 & SPC \\
B & 15.0 & 5.0 & 16.3 & 4.7 & SPC \\
C & 22.5 & 4.2 & 23.3 & 4.3 & SPC \\
D & 15.0 & 3.5 & 15.4 & 3.5 & SPC \\
E & 30.0 & 5.0 & 29.5 & 4.8 & SPC \\
F & 15.0 & 5.0 & 15.1 & 5.5 & SEPC \\
G & 30.0 & 3.5 & 32.0 & 3.7 & SEPC \\
H & 15.0 & 3.5 & 16.5 & 3.3 & SEPC \\
I & 30.0 & 5.0 & 30.8 & 6.4 & SEPC \\
J & 22.5 & 4.2 & 23.1 & 4.8 & SEPC \\
\hline
\end{tabular}


Table 3. Additional geometric and hydraulic characteristics of the meso-tube geometries tested

\begin{tabular}{|c|c|c|c|c|c|c|}
\hline $\begin{array}{c}\text { Tube } \\
\text { reference }\end{array}$ & $\begin{array}{c}\text { Orifice } \\
\text { open area, } \\
\alpha(\%)\end{array}$ & $\begin{array}{c}\text { Length to } \\
\text { diameter } \\
\text { ratio, } l / d \\
(-)\end{array}$ & $\begin{array}{l}\text { Internal } \\
\text { volume } \\
(\mathbf{m L})\end{array}$ & $\begin{array}{c}\text { No of } \\
\text { baffles per } \\
\text { unit length } \\
\left(\mathrm{m}^{-1}\right)\end{array}$ & $\begin{array}{c}\text { Relative } \\
\text { deviation } \\
\text { of } D_{c} / u L \text { fit } \\
(-)\end{array}$ & $\begin{array}{c}\text { Mean } \\
\text { hydraulic } \\
\text { time, } \\
\tau(\mathrm{s})\end{array}$ \\
\hline A & 0.12 & 3.0 & 58 & 33 & 0.39 & 160 \\
\hline B & 0.25 & 1.5 & 56 & 67 & 0.33 & \\
\hline $\mathrm{C}$ & 0.18 & 2.3 & 61 & 44 & 0.13 & 169 \\
\hline $\mathrm{D}$ & 0.12 & 1.5 & 48 & 67 & 0.83 & 133 \\
\hline $\mathrm{E}$ & 0.25 & 3.0 & 63 & 33 & 3 & 174 \\
\hline $\mathrm{F}$ & 0.25 & 1.5 & 69 & 67 & 0.24 & 191 \\
\hline G & 0.12 & 3.0 & 72 & 33 & 0.63 & 199 \\
\hline $\mathrm{H}$ & 0.12 & 1.5 & 54 & 67 & 0.30 & 149 \\
\hline I & 0.25 & 3.0 & 70 & 33 & 0.40 & 193 \\
\hline $\mathrm{J}$ & 0.18 & 2.3 & 65 & 44 & 0.25 & 180 \\
\hline
\end{tabular}

Table 4. Polynomial models for DoE contour plots shown in Figures 9 and 10

\section{Baffle shape}

\section{Polynomial model}

$$
D_{c}=\left(0.313 \alpha-0.038 \frac{l}{d}-0.070+0.238 \alpha \frac{l}{d}+1.530\right) \times 1 E-4
$$

SPC

$$
\frac{D_{c}}{u L}=\left(-0.375 \alpha+0.375 \frac{l}{d}-0.200+1.125 \alpha . T y p e(S P C)+5.200\right) \times 1 E-2
$$

$$
\frac{\bar{t}}{\tau}=-0.209 \alpha+0.015 \frac{l}{d}+0.057+1.494
$$

$$
D_{c}=\left(0.313 \alpha-0.038 \frac{l}{d}+0.070+0.238 \alpha \frac{l}{d}+1.530\right) \times 1 E-4
$$

SEPC

$$
\begin{gathered}
\frac{D_{c}}{u L}=\left(-0.375 \alpha+0.375 \frac{l}{d}+0.200-1.125 \alpha . \text { Type }(S E P C)+5.200\right) \times 1 E-2 \\
\frac{\bar{t}}{\tau}=-0.209 \alpha+0.015 \frac{l}{d}-0.057+1.494
\end{gathered}
$$


Table 5. Model parameters from the DoE including statistical significance for individual independent variables

\begin{tabular}{|c|c|c|c|c|}
\hline Model Parameters & Coefficient & $\begin{array}{l}\text { Standard } \\
\text { error }\end{array}$ & $\begin{array}{c}p \text {-Value (95\% } \\
\text { confidence) }\end{array}$ & $\begin{array}{l}\text { Confidence } \\
\text { interval }( \pm)\end{array}$ \\
\hline \multicolumn{5}{|l|}{ Response: $D_{c} / u L$} \\
\hline Constant & $5.20 \mathrm{E}-02$ & $1.39 \mathrm{E}-03$ & $1.78 \mathrm{E}-23$ & $2.86 \mathrm{E}-03$ \\
\hline$\alpha$ & $-3.75 \mathrm{E}-03$ & $1.55 \mathrm{E}-03$ & $2.34 \mathrm{E}-02$ & $3.20 \mathrm{E}-03$ \\
\hline$l / d$ & $3.75 \mathrm{E}-03$ & $1.55 \mathrm{E}-03$ & $2.32 \mathrm{E}-02$ & $3.20 \mathrm{E}-03$ \\
\hline Type(SPC) & $-2.00 \mathrm{E}-03$ & $1.39 \mathrm{E}-03$ & $1.62 \mathrm{E}-01$ & $2.86 \mathrm{E}-03$ \\
\hline Type(SEPC) & $2.00 \mathrm{E}-03$ & $1.39 \mathrm{E}-03$ & $1.62 \mathrm{E}-01$ & $2.86 \mathrm{E}-03$ \\
\hline$\alpha^{* T y p e(S P C)}$ & $1.13 \mathrm{E}-02$ & $1.55 \mathrm{E}-03$ & $1.37 \mathrm{E}-07$ & $3.20 \mathrm{E}-03$ \\
\hline$\alpha *$ Type(SEPC) & $-1.13 \mathrm{E}-02$ & $1.55 \mathrm{E}-03$ & $1.37 \mathrm{E}-07$ & $3.20 \mathrm{E}-03$ \\
\hline \multicolumn{5}{|l|}{ Response: $\mathbf{D}_{\mathrm{c}}$} \\
\hline Constant & $1.53 \mathrm{E}+00$ & $2.59 \mathrm{E}-02$ & $2.33 \mathrm{E}-28$ & $5.34 \mathrm{E}-02$ \\
\hline$\alpha$ & $3.13 \mathrm{E}-01$ & $2.90 \mathrm{E}-02$ & $6.97 \mathrm{E}-11$ & $5.97 \mathrm{E}-02$ \\
\hline$l / d$ & $-3.75 E-02$ & $2.90 \mathrm{E}-02$ & $2.08 \mathrm{E}-01$ & $5.97 \mathrm{E}-02$ \\
\hline Type(SPC) & $-7.00 \mathrm{E}-02$ & $2.59 \mathrm{E}-02$ & $1.23 \mathrm{E}-02$ & $5.34 \mathrm{E}-02$ \\
\hline Type(SEPC) & $7.00 \mathrm{E}-02$ & $2.59 \mathrm{E}-02$ & $1.23 \mathrm{E}-02$ & $5.34 \mathrm{E}-02$ \\
\hline$\alpha * l / d$ & $2.38 \mathrm{E}-01$ & $2.90 \mathrm{E}-02$ & $1.53 \mathrm{E}-08$ & $5.97 \mathrm{E}-02$ \\
\hline \multicolumn{5}{|l|}{ Response: $\bar{t} / \tau$} \\
\hline Constant & $1.49 \mathrm{E}+00$ & $1.09 \mathrm{E}-02$ & $0.00 \mathrm{E}+00$ & $2.23 \mathrm{E}-02$ \\
\hline$\alpha$ & $-2.09 \mathrm{E}-01$ & $1.22 \mathrm{E}-02$ & $9.72 \mathrm{E}-16$ & $2.50 \mathrm{E}-02$ \\
\hline$l / d$ & & $1.22 \mathrm{E}-02$ & $2.36 \mathrm{E}-01$ & $2.50 \mathrm{E}-02$ \\
\hline Type(SPC) & $5.67 \mathrm{E}-02$ & $1.09 \mathrm{E}-02$ & $1.89 \mathrm{E}-05$ & $2.23 \mathrm{E}-02$ \\
\hline Type(SEPC) & $-5.67 \mathrm{E}-02$ & $1.09 \mathrm{E}-02$ & $1.89 \mathrm{E}-05$ & $2.23 \mathrm{E}-02$ \\
\hline
\end{tabular}




\section{Figure captions}

Figure 1. Internal geometries of the SPC (A-E) and SEPC (F-J) meso-tubes tested. The inner diameter of the tubes was fixed at $d=10 \mathrm{~mm}$, and baffle spacing and orifice diameter varied as detailed in Table 2. The actual dimensions of the baffled sections of the meso-tubes shown in the microphotographs are summarised in Table 2.

Figure 2. Experimental setup used for the batch and continuous solid RTD studies.

Figure 3. Suspension of PVC particles in tube $A$ (SPC) and tube $G$ ( SEPC) showing (top view): (a)-(b) the settled particles in the tube without oscillation; (c)-(d) the onset of oscillation; and (e)-(f) eddies propagation in fully suspended particles.

Figure 4. Batch suspension of PVC particles in SPC and SPEC meso-tubes. (a) Batch suspension studies showing critical (minimum) fluid oscillation amplitude, $x_{o, \text { crit }}$ for full suspension of particles. (b) Corresponding oscillation peak velocity, $u(t)_{\max }$ required for full suspension of particles.

Figure 5. Characteristics of PVC particles used in this study. (a)-(b) Size distribution of particles using QICPIC image analysis instrument. (c) Distribution of settling velocity estimated for PVC particles based on Stokes's law and particle sizes from QICPIC.

Figure 6. Relationship between power input, $P / V$ and critical amplitude, $x_{o, c r i t}$ for full batch suspension of PVC particles. (a) Effect of $x_{0, c r i t}$ on power density, $P / V$ (b) Effect of $\alpha$ on $x_{0, \text { crit }}$.

Figure 7. $F(\theta)$-curves for continuous liquid-solid RTD experiments in: (a) SPC and (b) SEPC meso-tubes.

Figure 8. Best-fitting of experimental solids RTD with plug flow with axial dispersion model for SPC and SEPC meso-tubes. Effect of meso-tube geometry on (a) dimensionless axial dispersion coefficient, $D_{c} / u L$, (b) axial dispersion coefficient, $D_{c}$ and (c) model mean residence time, $t_{m}$.

Figure 9. Surface DoE plots showing the interaction between key geometrical parameters for SPC and SEPC baffle types. (a) Effect of $\alpha$ and $l / d$ ratio on axial dispersion coefficient, $D_{c}$ $\left(\mathrm{m}^{2} \mathrm{~s}^{-1}\right)$ (b) Effect of $\alpha$ and $l / d$ ratio on dimensionless axial dispersion coefficient, $D d u L$.

Figure 10. (a) Comparison of the experimental mean residence time, $\bar{t}(\mathrm{~s})$ and hydraulic time, $\tau$ for continuous liquid-solid RTD (b) Comparison of best-fitted residence time, $t_{m}$ from the plug flow with axial dispersion model with $\bar{t}(\mathrm{~s})$. (c) Surface DoE plots showing the interaction between $\alpha, l / d$ and ratio of mean experimental solids residence time to hydraulic time, $\bar{t} / \tau$ for SPC and SEPC baffled tubes. 


\section{List of Figures}
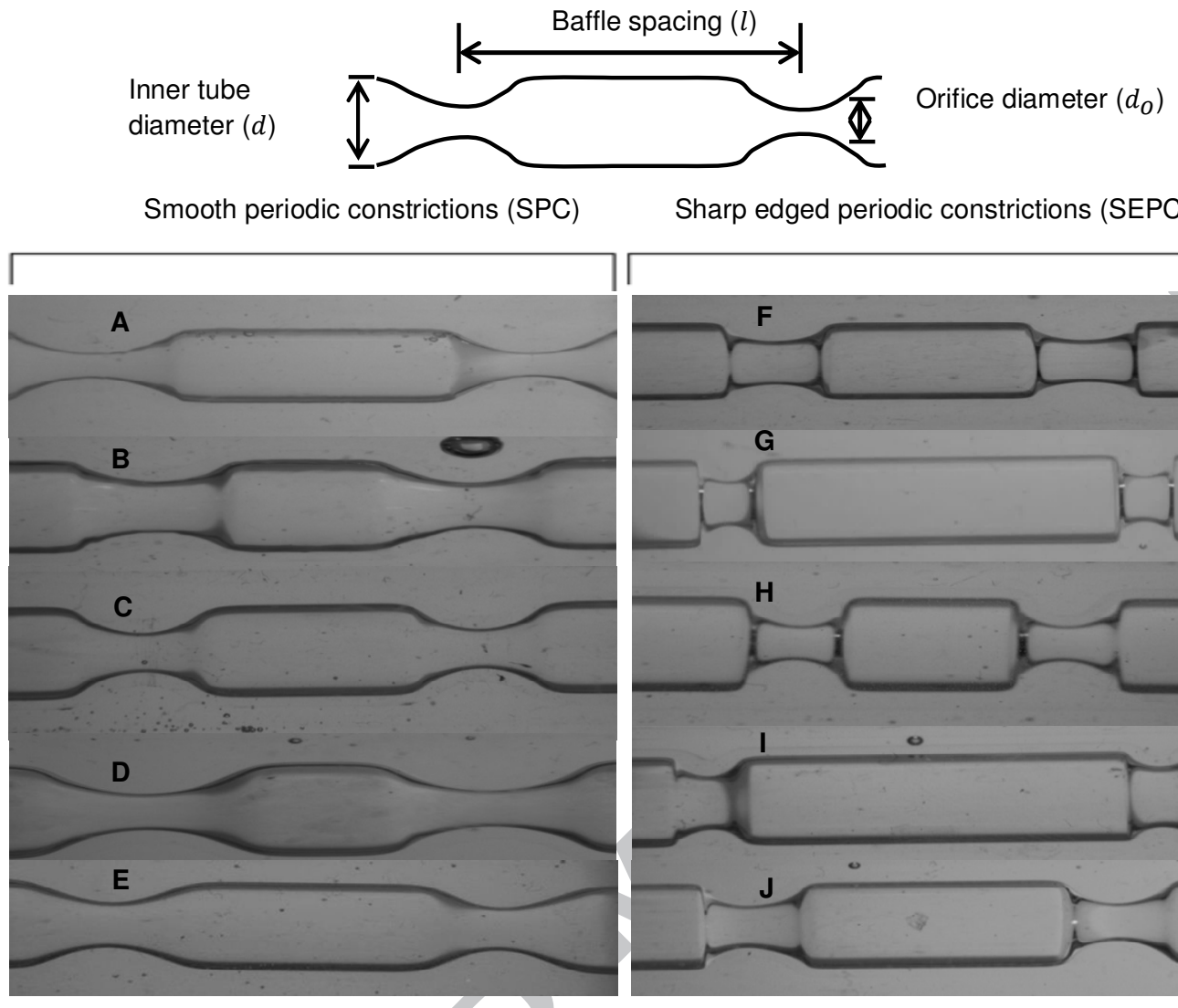

\section{Sharp edged periodic constrictions (SEPC)}

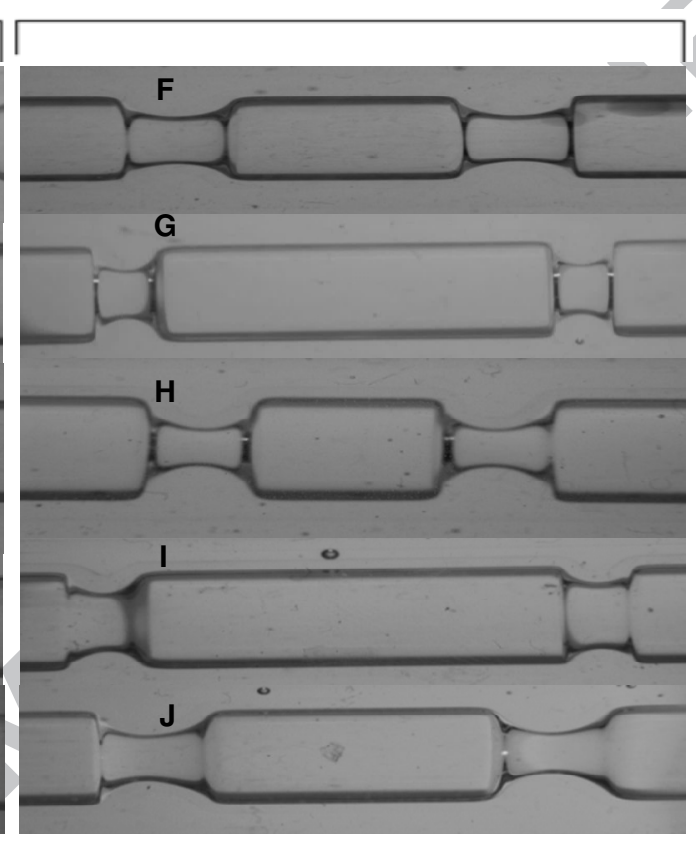

Figure 1 


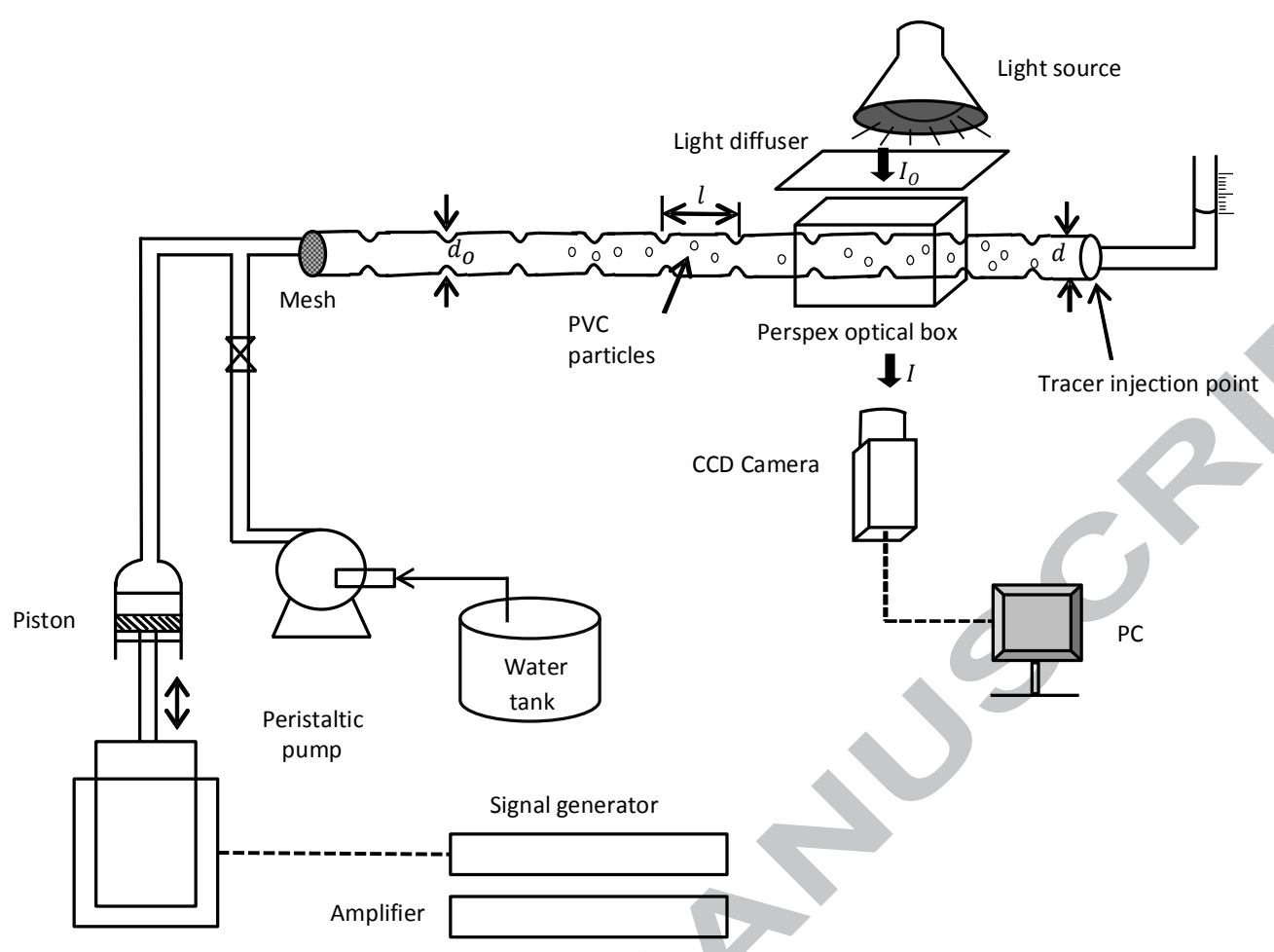

Figure 2 
Tube A (SPC)

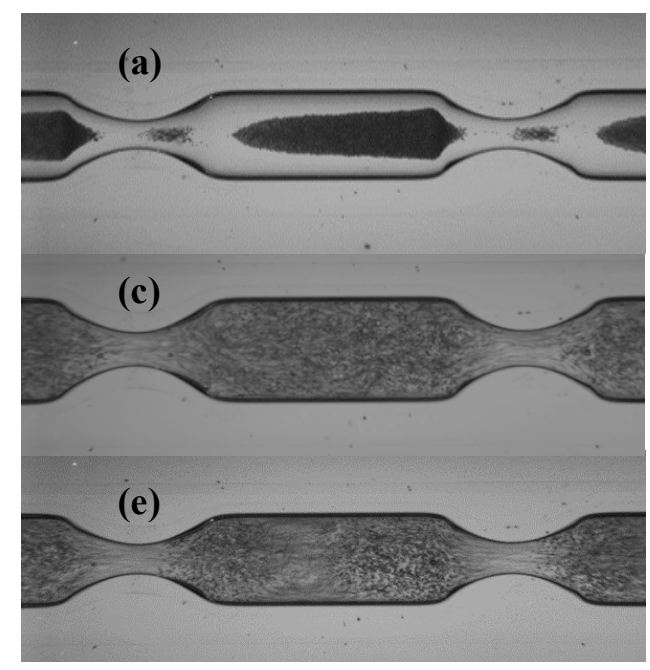

Tube G (SEPC)

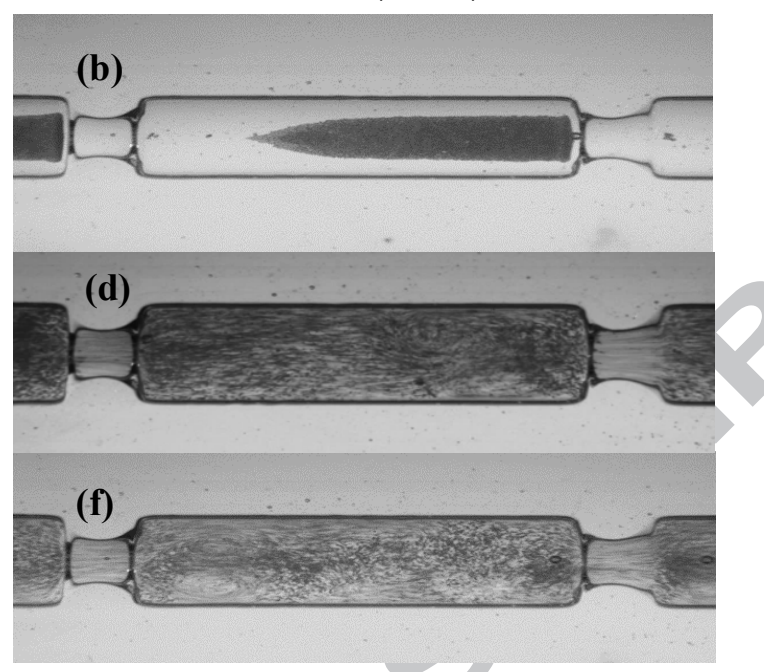

Figure 3 


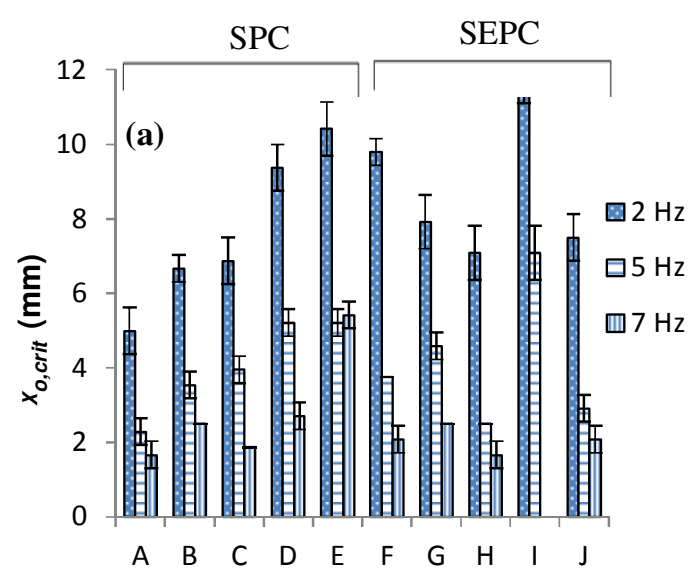

Meso-tube geometry

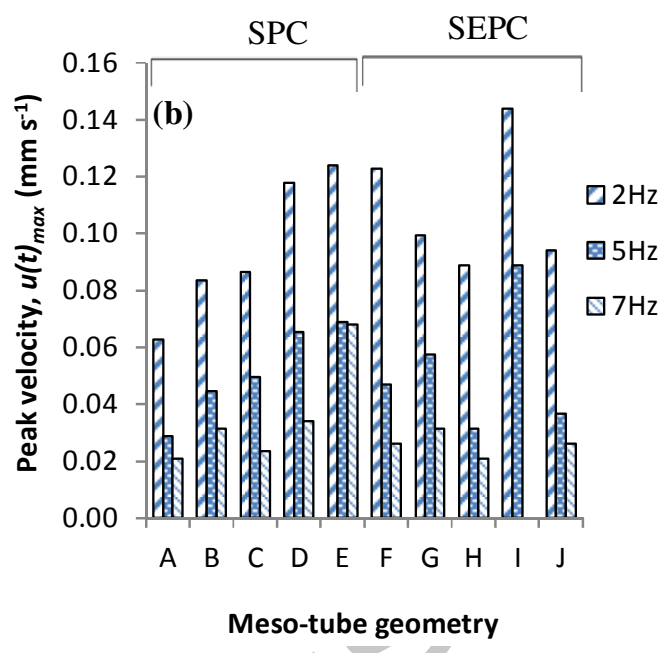

Figure 4 

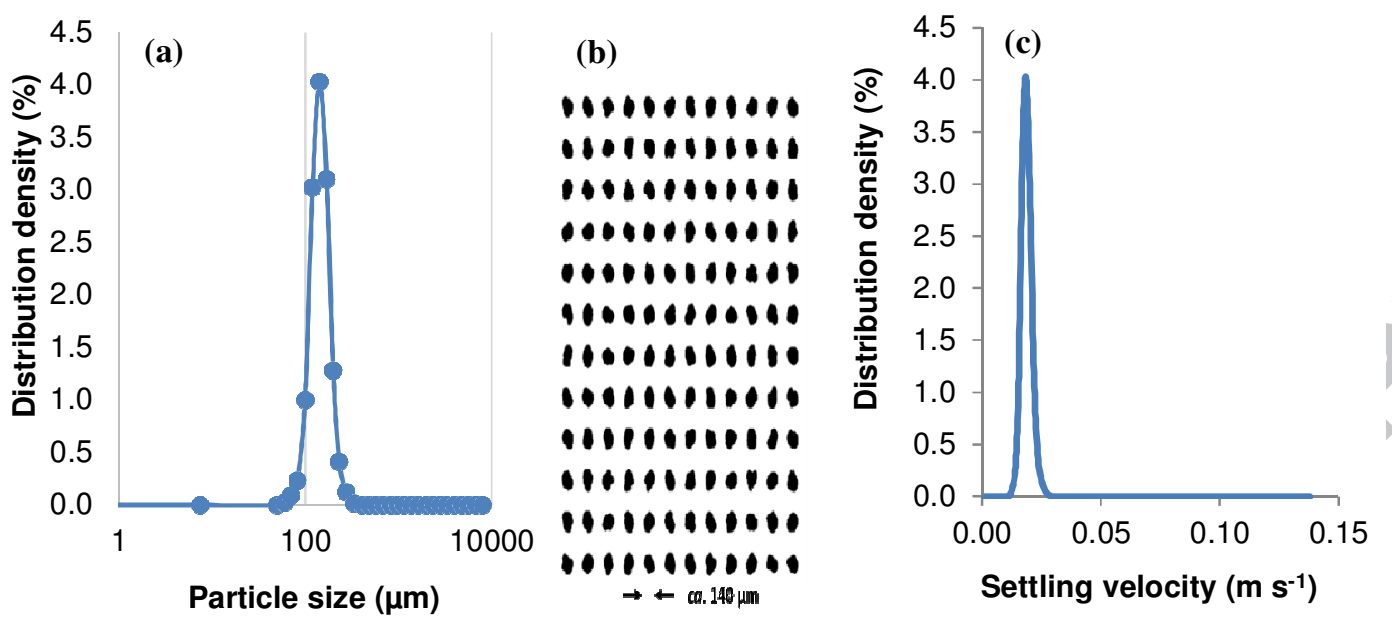

Figure 5 


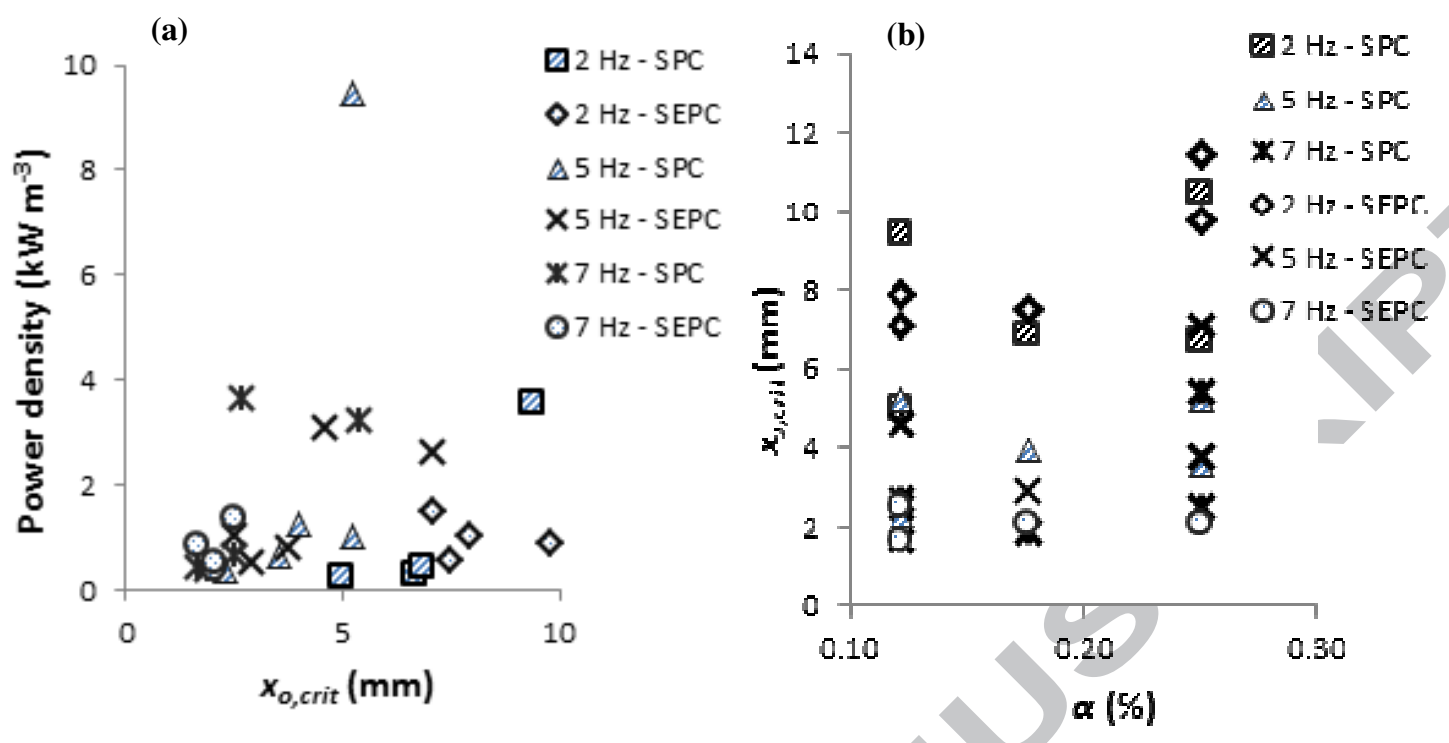

Figure 6 

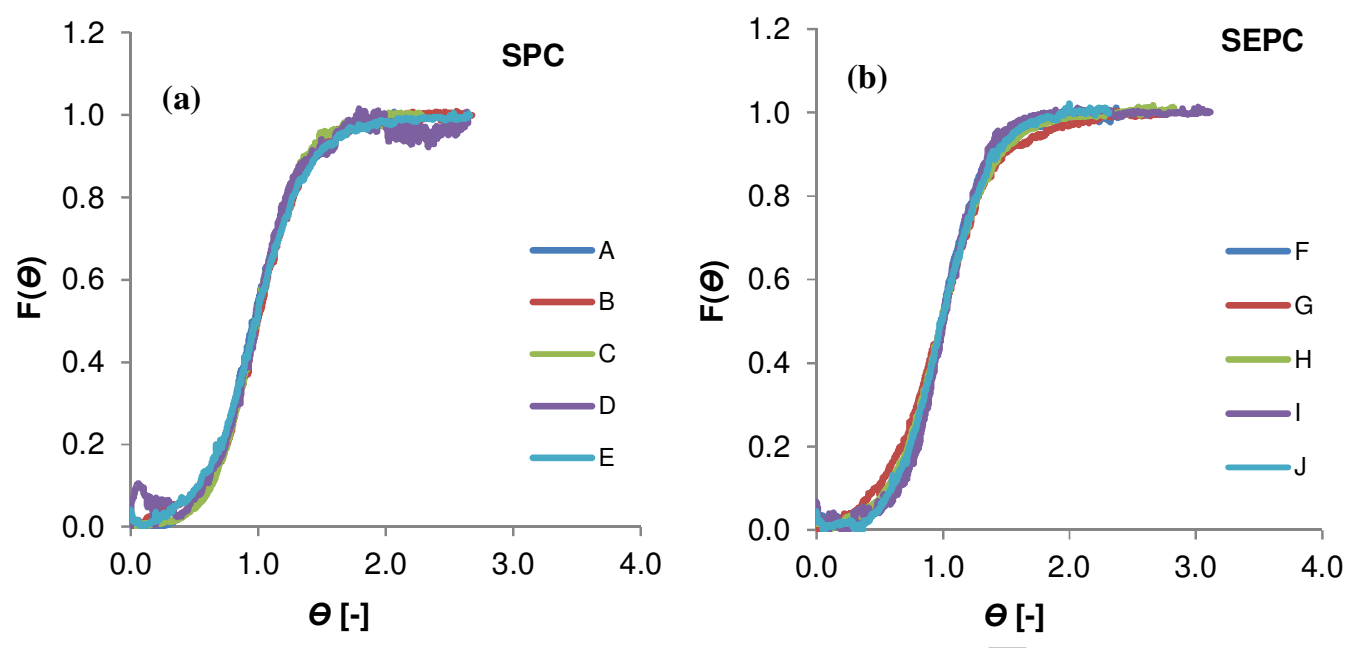

Figure 7 


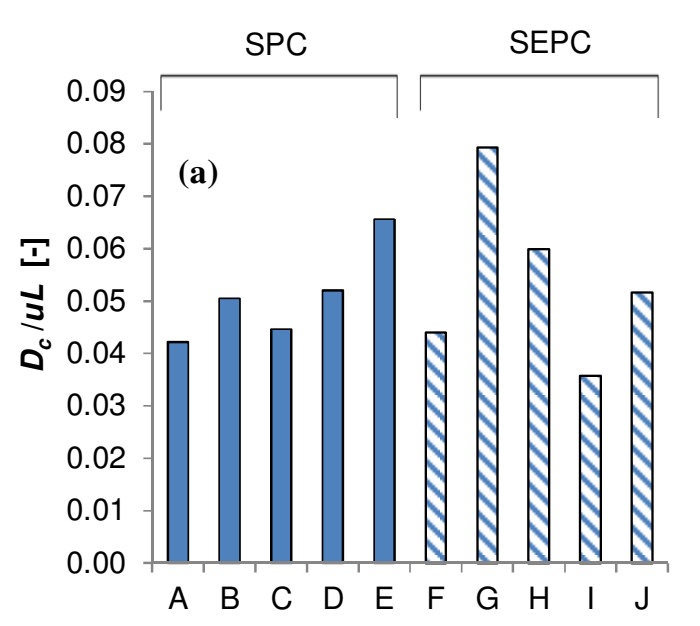

Meso-tube geometry
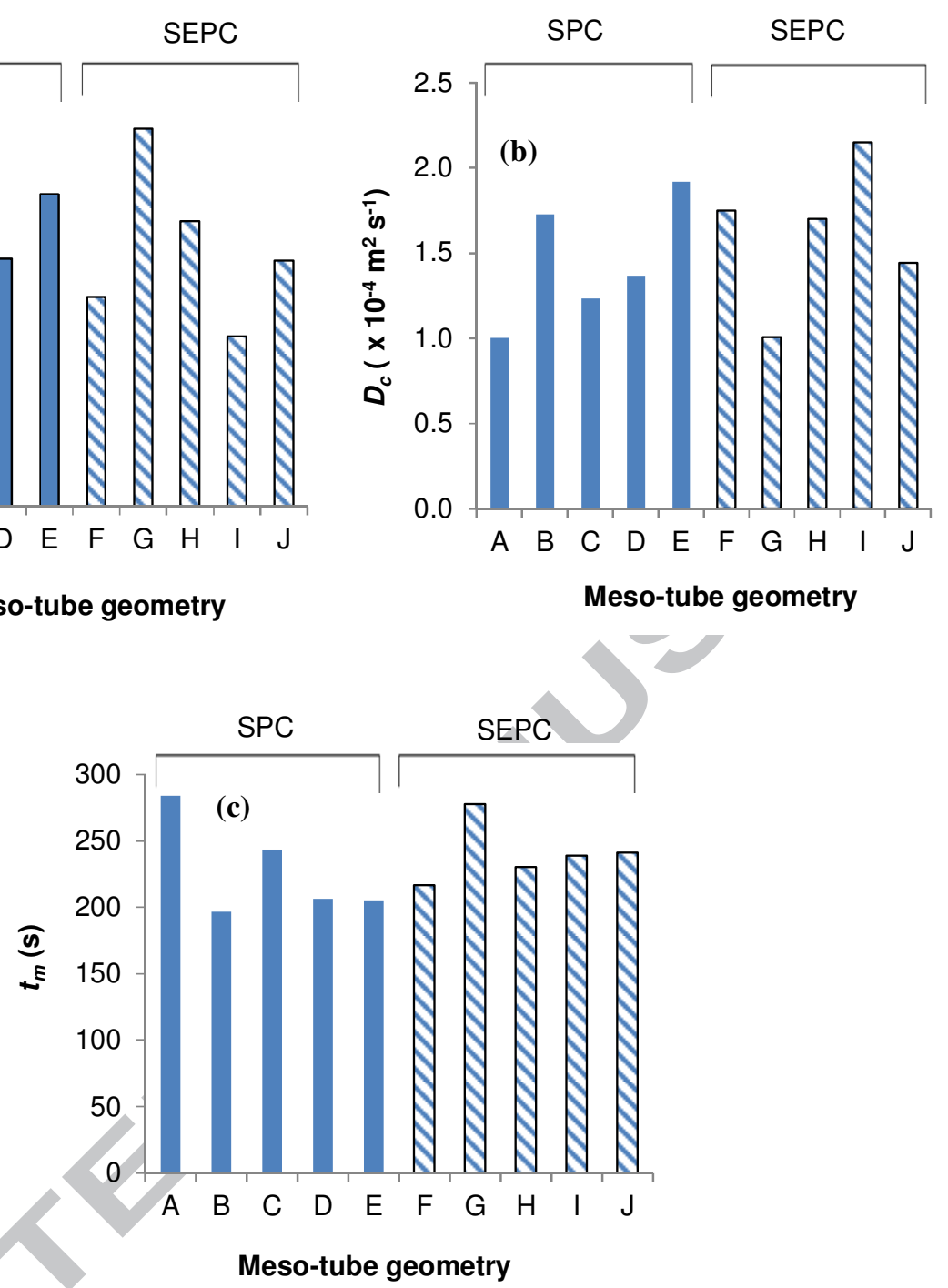

Figure 8 

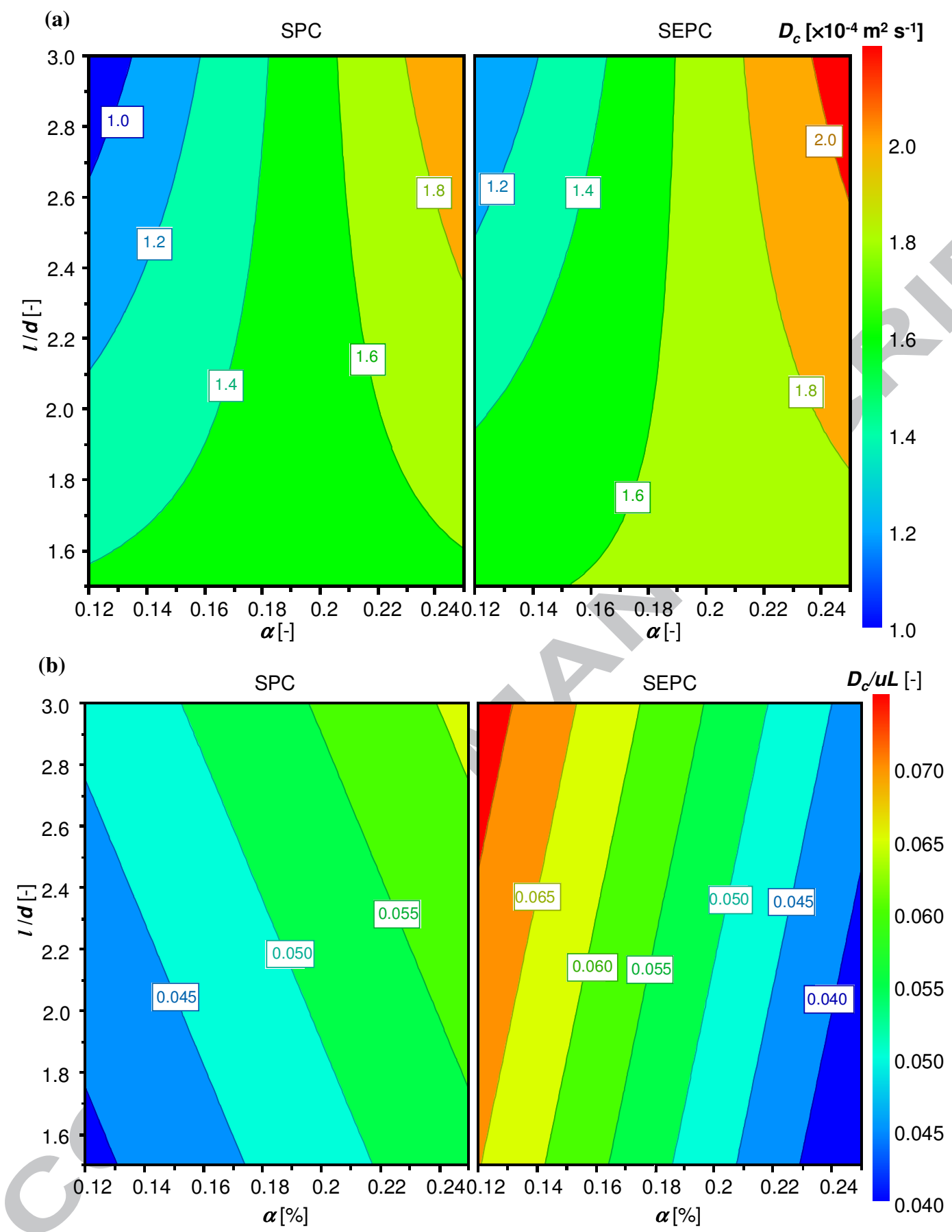

Figure 9 

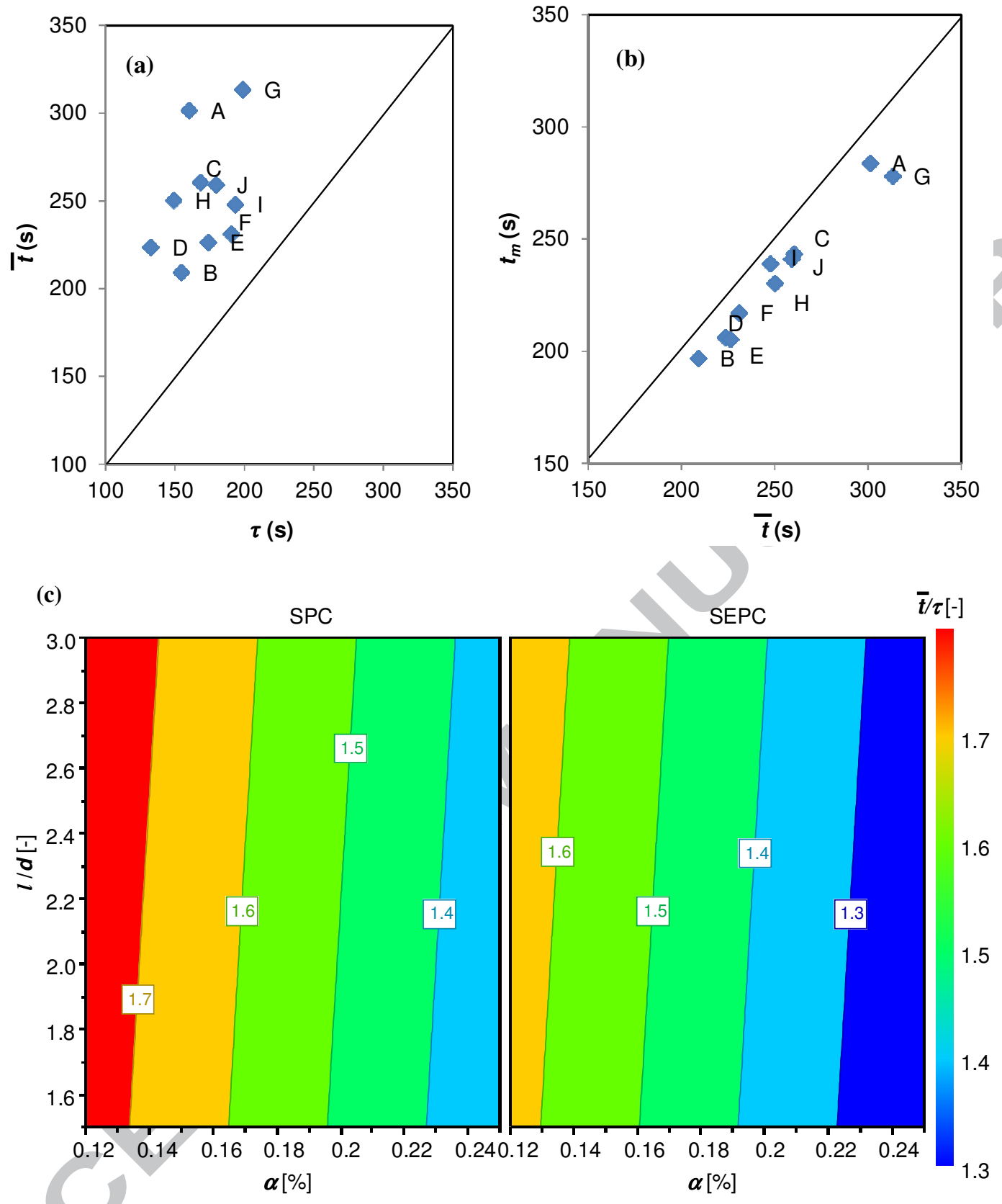

Figure 10 


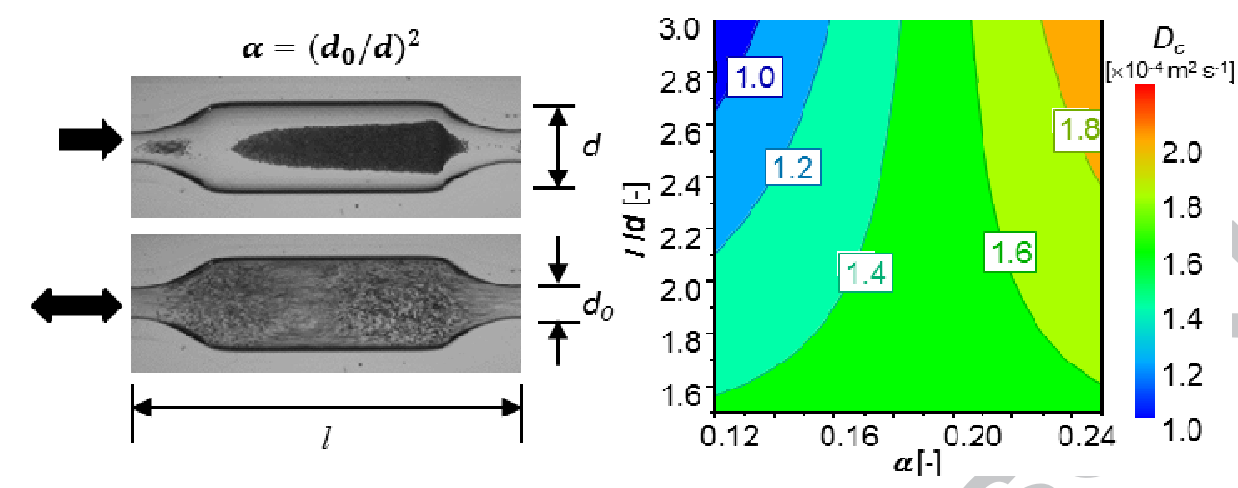




\section{Highlights:}

- The effect of inner geometry of meso-scale OFRs on axial dispersion was studied

- The impact on batch suspension and continuous liquid-solid flows was studied

- The effect of $d_{o}, \alpha, l$ and baffle shape was evaluated with a $2 \times 2$ factorial design

- Continuous liquid-solid flow modelled with plug flow with axial dispersion model

- Smooth-edged constrictions produced sharper solids RTDs 